\title{
An Intrinsic Behavioural Approach to the Gap Metric
}

\author{
Wenming Bian, $\quad$ Mark French ${ }^{\dagger} \quad$ Harish K. Pillai ${ }^{\ddagger}$ \\ March 8th 2006 \\ Submitted to SIAM Journal of Control and Optimization
}

\begin{abstract}
An intrinsic trajectory level approach without any recourse to an algebraic structure of a representation is utilized to develop a behavioural approach to robust stability. In particular it is shown how the controllable behaviour can be constructed at the trajectory level via Zorn's Lemma, and this is utilized to study the controllable-autonomous decomposition. Stability concepts are defined and the relation between this framework and the well known difficulties of classical input-output approaches to systems over the doubly infinite time-axis are discussed. The gap distance is generalised to the behavioural setting via a trajectory level definition; and a basic robust stability theorem is established for linear shift invariant behaviours. The robust stability theorem is shown to provide an explicit robustness interpretation to the behavioural $\mathcal{H}^{\infty}$ synthesis of Willems and Trentelmann.
\end{abstract}

\section{Introduction}

We begin by observing that the graph topology with its various metrizations plays a fundamental role in the theory of robust stability for classical LTI systems $([1,4,20])$. The contribution of this note is to develop the basic theory of robust stability involving the gap-distance directly from a behavioural perspective, observing that recent approaches to generalisations of the gap metric [4] have been purely trajectory based and hence are easily amenable to such a approach. There has been previous interest in developing behavioural notions of the gap metric, see e.g. [11] for an example.

From a behavioural point of view $([7,14,15,16])$, the approach is especially fundamental. Much has been made of the intrinsic nature of behavioural definitions and the need for 'representation free' approaches. In this note, we do not recourse to representations at all, indeed all proofs are at the intrinsic trajectory level and are not restricted, for example to differential systems. This gives this paper a different 'flavour' to much of the recent behavioural literature which is predominately of an algebraic nature. We illustrate our results by considering a system with a pair of (in general) non-commensurate delays: such a system class falls out of the scope of the existing algebraic techniques of the existing behavioural theory for delay systems where to achieve an algebraic structure, delays are assumed to be commensurate [5].

\footnotetext{
${ }^{*}$ School of Electronics and Computer Science, University of Southampton, SO17 1BJ, UK, wb@ecs.soton.ac.uk

${ }^{\dagger}$ School of Electronics and Computer Science, University of Southampton, SO17 1BJ, UK, mcf@ecs.soton.ac.uk,

${ }^{\ddagger}$ Department of Electrical Engineering, Indian Institute of Technology, Bombay, India hp@ee.iitb.ac.in.
} 
There are two inter-related reasons for this approach. The first is mathematical: only a limited set-theoretic/analytic structure is required to obtain the required results; hence it is inappropriate to utilize any further structures (e.g. of an algebraic type); this in turn yields greater generality. The second is of applied consequence: a powerful robust stability result should impose as little structure as possible on the structure of the perturbations permitted: the perturbed systems may not be representable by differential systems; and may for example arise as a delay or distributed system, or even defy direct representation. A set theoretic treatment of the perturbed system is therefore the appropriate treatment.

Our basic robust stability theorem provides a self-contained basis for the robustness interpretation of the behavioural $\mathcal{H}^{\infty}$ results in $[17,12]$. The composition of the set-theoretic/analytic treatment in this paper of the resulting perturbed systems is thus set against the controller synthesis for the nominal system, which appropriately is in the context of systems with greater structure (i.e. differential systems).

In relation to the classical approaches, we remark that the standard $\mathcal{H}^{2}$ gap is a metric on transfer functions, and does not directly apply to systems which either have non-zero initial conditions or which are not minimal (i.e. have non-controllable modes). The $\nu$-gap ([13]) metric also induces the graph topology on transfer functions, and can handle non-zero initial conditions at zero by its intrinsic definition on the doubly infinite time-axis. However, the $\nu$-gap is also only directly applicable to controllable systems. By defining systems to be limits of Cauchy sequences in the graph topology [13], the standard gap approaches can also be extended to non-minimal cases; a contribution of this paper from a classical perspective is to provide an alternate and slightly more general approach to these cases. The trajectory formulation considered is also directly applicable to infinite dimensional systems both in the context of the nominal and perturbed plant and controller: for example delay-differential systems are directly handled, compare to the less direct classical techniques (e.g. the Cauchy sequence approach). We observe also that within the classical framework there has been a move towards representation free approaches to the gap, e.g. especially for approaches to nonlinear systems [4]. The behavioural approach considered here is one natural extension of this viewpoint.

We emphasize that the main contribution is not in the minor increase in scope of the resulting theorem (as discussed above), rather the contribution is in an alternative and direct derivation of the results within a behavioural framework. Furthermore we argue that the behavioural approach taken overcomes the well known difficulties [3] of input-output systems theories on the doubly infinite time axis.

\section{Behavioural Definitions}

Let $\mathcal{T}$ denote the time set, taken throughout to be either $\mathbb{Z}$ or $\mathbb{R}$, and let $\mathcal{T}_{+}=\mathbb{N}$ if $\mathcal{T}=\mathbb{Z}$ and $\mathcal{T}_{+}=\mathbb{R}_{+}$if $\mathcal{T}=\mathbb{R}$. An interval, say $[a, b]$, is understood as $[a, b]=\{t \in \mathcal{T}, a \leq t \leq b\}$. For $n \geq 1$, let $\operatorname{map}\left(\mathcal{T}, \mathbb{R}^{n}\right)$ be the set of all maps from $\mathcal{T}$ to $\mathbb{R}^{n}$. A $n$-valued behaviour $\mathfrak{B}$ is a subset of $\operatorname{map}\left(\mathcal{T}, \mathbb{R}^{n}\right)$, i.e. $\mathfrak{B} \subset \operatorname{map}\left(\mathcal{T}, \mathbb{R}^{n}\right)$. The shift operator $\sigma_{t}, t \in \mathcal{T}$ is defined: $\sigma_{t} w(\cdot)=w(\cdot+t)$.

Definition 2.1 Let $\mathfrak{B}$ be a behaviour. Then:

1. $\mathfrak{B}$ is said to be linear if $\mathfrak{B}$ is a vector space.

2. $\mathfrak{B}$ is said to be shift invariant (time invariant) if $w \in \mathfrak{B}$ implies $\sigma_{t} w \in \mathfrak{B}$ for all $t \in \mathcal{T}$.

Smooth differential behaviours are linear, shift invariant, continuous-time behaviours which can be expressed as the kernel of a differential operator, ie. those for which there exists a polynomial 
valued matrix $R$ s.t. that

$$
\mathfrak{B}=\left\{w \in C^{\infty} \mid R\left(\frac{d}{d t}\right) w=0\right\} .
$$

Equivalently in the discrete-time setting, the operator is that of unit shifts:

$$
\mathfrak{B}=\left\{w \in \operatorname{map}\left(\mathbb{Z}, \mathbb{R}^{n}\right) \mid R\left(\sigma_{1}\right) w=0\right\} .
$$

and such behaviours are called difference behaviours.

Observe that in this note we will be interested in non-differential/difference behaviours, for example, systems incorporating a time delay.

Definition 2.2 A behaviour $\mathfrak{B}$ is said to have memory $l \geq 0$ if for any $w_{1}, w_{2} \in \mathfrak{B}$ with $\left.w_{1}\right|_{[a, a+l]}=\left.w_{2}\right|_{[a, a+l]}$ and $a \in \mathcal{T}$, the trajectory

$$
w_{3}(t)= \begin{cases}w_{1}(t) & \text { if } t \leq a, \\ w_{2}(t) & \text { if } t \geq a\end{cases}
$$

also lies in $\mathfrak{B}$.

Clearly, a shift invariant behaviour $\mathfrak{B}$ has memory $l \geq 0$ if and only if for any $w_{1}, w_{2} \in \mathfrak{B}$ with $\left.w_{1}\right|_{[0, l]}=\left.w_{2}\right|_{[0, l]}$, the trajectory

$$
w_{3}(t)= \begin{cases}w_{1}(t) & \text { if } t \leq 0 \\ w_{2}(t) & \text { if } t \geq 0\end{cases}
$$

also lies in $\mathfrak{B}$.

If a behaviour has memory $0 \leq l<\infty$, it is said to have finite memory, if $l=0$ then it is memoryless. Note that a non-memoryless continuous time differential behaviour has finite memory, and $l>0$ can be taken to be arbitrarily small; a discrete time behaviour also has finite memory, and here $l \geq 0$ depends on the system order. The minimal memory $l_{0} \geq 0$ of a behaviour $\mathfrak{B}$ is the largest number s.t. $\mathfrak{B}$ has memory $l$ for all $l>l_{0}$. Note that the minimum is not necessarily attained.

The standard definition of autonomy is that behaviour $\mathfrak{B}$ is said to be autonomous if for any $w_{1}, w_{2} \in \mathfrak{B},\left.w_{1}\right|_{(-\infty, 0]}=\left.w_{2}\right|_{(-\infty, 0]}$ implies $w_{1}=w_{2}$. Note that as far as differential systems are concerned, autonomous behaviours have finite memory. We therefore relax the definition for autonomy as follows:

Definition 2.3 A behaviour $\mathfrak{B}$ is said autonomous if there exists $0 \leq l_{0}<\infty$ such that for any $w_{1}, w_{2} \in \mathfrak{B}$ and any interval $V$ of length greater than $l_{0},\left.w_{1}\right|_{V}=\left.w_{2}\right|_{V}$ implies $w_{1}=w_{2}$.

Non-autonomy of a behaviour is thus just the existence of a trajectory in the behaviour whose support has complement containing an interval of length greater than $l_{0}$, eg. a compactly supported trajectory.

It should be observed that it is possible that if $\mathfrak{B}$ has minimal memory $l_{0}$, then e.g. an autonomous sub-behaviour can have a minimal memory i) $l=0$, ii) $0<l<l_{0}$, or iii) $l=l_{0}$, for example consider:

$$
\dot{y}(t)=a y(t-2 \tau)+b y(t-\tau)+c y(t)+d u(t-2 \tau),
$$

then $l_{0}=2 \tau$ and the autonomous behaviour $\mathfrak{B}_{u=0}$ corresponds to i) if $a=b=0$, ii) if $a=c=0, b \neq 0$, and iii) if $b=c=0, a \neq 0$. 
The behavioural notion of controllability is defined in [7] for differential hehaviours. However, this definition is restrictive since it assumes shift-invariance of the behaviour concerned. We therefore give a modified definition for behavioural controllability that is applicable to more general beaviours but coincides with the notion of controllability in [7] for the case of shiftinvariant behaviours.

Definition 2.4 A behaviour $\mathfrak{B}$ is said to be controllable if given $w_{1}, w_{2} \in \mathfrak{B}$ and $s \in \mathcal{T}$, there exist $w_{3} \in \mathfrak{B}$ and $\tau \in \mathcal{T}_{+}$such that

$$
w_{3}(t)= \begin{cases}w_{1}(t) & \text { if } t \leq s \\ w_{2}(t) & \text { if } t \geq s+\tau .\end{cases}
$$

This definition requires that the concatenating function $w_{3}$ lies in $\mathfrak{B}$. This can be hard to guarantee in certain cases including generalisations to multi-dimensional systems. Therefore we next introduce the notion of $\mathfrak{B}$-controllability.

Definition 2.5 Given a behaviour $\mathfrak{B}$, a sub-behaviour $\mathfrak{B}^{*} \subset \mathfrak{B}$ is said to be $\mathfrak{B}$-controllable if for all $w_{1}, w_{2} \in \mathfrak{B}^{*}$ and $s \in \mathcal{T}$ there exist $w_{3} \in \mathfrak{B}$ and $\tau \in \mathcal{T}_{+}$such that

$$
w_{3}(t)= \begin{cases}w_{1}(t) & \text { if } t \leq s, \\ w_{2}(t) & \text { if } t \geq s+\tau .\end{cases}
$$

We remark that if $\mathfrak{B}, \mathfrak{B}^{*}$ are both shift invariant behaviours and $\mathfrak{B}^{*} \subset \mathfrak{B}$, then $\mathfrak{B}^{*}$ is $\mathfrak{B}$ controllable if and only if given any $w_{1}, w_{2} \in \mathfrak{B}^{*}$, there exists $w_{3} \in \mathfrak{B}$ and $\tau \in \mathcal{T}_{+}$such that

$$
w_{3}(t)= \begin{cases}w_{1}(t) & \text { if } t \leq 0, \\ w_{2}(t) & \text { if } t \geq \tau .\end{cases}
$$

If $\mathfrak{B}^{*}=\mathfrak{B}$, then the $\mathfrak{B}$-controllability of $\mathfrak{B}^{*}$ is the same as controllability defined by Definition 2.4. So controllability in the sense of Definition 2.4 implies $\mathfrak{B}$-controllability. But the following example shows that the converse does not hold.

Example 2.6 Let $\mathfrak{B}=C^{\infty}(\mathbb{R}, \mathbb{R})$ and $\mathfrak{B}^{*}$ be the set of all constant functions. Then both $\mathfrak{B}$ and $\mathfrak{B}^{*}$ are linear shift invariant behaviours with finite memory and $\mathfrak{B}^{*} \subset \mathfrak{B}$. It is straightforward to check that $\mathfrak{B}^{*}$ is $\mathfrak{B}$-controllable. However, it is neither $\mathfrak{B}^{*}$-controllable nor controllable in the sense of Definition 2.4. Similarly, let $\mathfrak{B}=C^{0}(\mathbb{R}, \mathbb{R})$. Then the sub-behaviour $\mathfrak{B}^{*}=\operatorname{span}\left\{e^{t}\right\}$ is $\mathfrak{B}$-controllable but it is not $\mathfrak{B}^{*}$-controllable.

We now consider the properties of $\mathfrak{B}$-controllable behaviours.

Lemma 2.7 Suppose $\mathfrak{B}$ is a behaviour. Then there exists at least one maximal $\mathfrak{B}$-controllable sub-behaviour.

Proof. Firstly, any behaviour has at least one $\mathfrak{B}$-controllable subbehaviour, i.e. $\{0\}$. Secondly, set inclusion defines a partial order on the set of all $\mathfrak{B}$-controllable sub-behaviours. Any chain of $\mathfrak{B}$-controllable sub-behaviours:

$$
\mathfrak{B}_{\alpha} \subset \mathfrak{B}_{\beta} \subset \cdots \subset \mathfrak{B}_{\gamma} \subset \ldots
$$

where $\alpha, \beta, \gamma \cdots \in \Gamma$ and $\Gamma$ the index set, has an upper bound:

$$
\mathfrak{B}_{\alpha} \subset \cup_{\beta \in \Gamma} \mathfrak{B}_{\beta}=\mathfrak{B}^{*} .
$$

$\mathfrak{B}^{*} \subset \mathfrak{B}$ is $\mathfrak{B}$-controllable since given any $w_{1}, w_{2} \in \mathfrak{B}^{*}$, we have $w_{1} \in B_{\alpha}, w_{2} \in \mathfrak{B}_{\beta}$ for some $\alpha, \beta \in \Gamma$, hence $w_{1}, w_{2} \in \mathfrak{B}_{\gamma}, \gamma=\max \{\alpha, \beta\}$, and by the $\mathfrak{B}$-controllability of $\mathfrak{B}_{\gamma}$ it follows that there exists $w_{3} \in \mathfrak{B}$ satisfying equation (2.6) as required. Zorn's lemma then gives the existence of a maximal $\mathfrak{B}$-controllable sub-behaviour as required. 
Note that this set-theoretic construction is extremely general: we do not require any linearity, memory or differential/difference structure on $\mathfrak{B}$. In general, maximal $\mathfrak{B}$-controllable subbehaviours are not unique. However, if the behaviour $\mathfrak{B}$ is linear, then there exists a unique maximal $\mathfrak{B}$-controllable linear sub-behaviour.

Theorem 2.8 Suppose $\mathfrak{B}$ is a linear behaviour. Then there exists a unique maximal linear $\mathfrak{B}$-controllable sub-behaviour.

Proof. We consider the set of all linear $\mathfrak{B}$-controllable sub-behaviours. With the relation induced by subset inclusion, this set is partially ordered and a maximal sub-behaviour $\mathfrak{B}_{\text {cont }}$ exists which is also linear.

To show the uniqueness, let $\mathfrak{B}_{1}$ be another linear maximal $\mathfrak{B}$-controllable sub-behaviour and let $\mathfrak{B}_{2}$ denote the linear span of $\mathfrak{B}_{\text {cont }}$ and $\mathfrak{B}_{1}: \mathfrak{B}_{2}=\mathfrak{B}_{\text {cont }}+\mathfrak{B}_{1}$. Let $w_{1}, w_{2} \in \mathfrak{B}_{2}$. Without loss of generality, we may suppose that $w_{i}=\alpha_{i} x_{i}+\beta_{i} y_{i}$ with $\alpha_{i}, \beta_{i} \in \mathbb{R}, x_{i} \in \mathfrak{B}_{\text {cont }}, y_{i} \in \mathfrak{B}_{1}$ and $i=1,2$. Since $0 \in \mathfrak{B}_{\text {cont }} \cap \mathfrak{B}_{1}$ and by the definition of $\mathfrak{B}$-controllability, we have: for all $s \in \mathcal{T}$, there exist $\tau_{1}, \tau_{2}>0$ and $z_{1}, v_{1} \in \mathfrak{B}$ such that $\left.z_{1}\right|_{(-\infty, s]}=\left.x_{1}\right|_{(-\infty, s]},\left.z_{1}\right|_{\left[s+\tau_{1}, \infty\right)}=\left.0\right|_{\left[s+\tau_{1}, \infty\right)},\left.v_{1}\right|_{(-\infty, s]}=$ $\left.y_{1}\right|_{(-\infty, s]},\left.v_{1}\right|_{\left[s+\tau_{2}, \infty\right)}=\left.0\right|_{\left[s+\tau_{2}, \infty\right)}$. Let $\tau_{3}=\max \left\{\tau_{1}, \tau_{2}\right\}$ and $w_{3}=\alpha_{1} z_{1}+\beta_{1} v_{1} \in \mathfrak{B}$. Then we have $\left.w_{3}\right|_{(-\infty, s]}=\left.\alpha_{1} x_{1}\right|_{(-\infty, s]}+\left.\beta_{1} y_{1}\right|_{(-\infty, s]}=\left.w_{1}\right|_{(-\infty, s]}$ and $\left.w_{3}\right|_{\left[s+\tau_{3}, \infty\right)}=\left.0\right|_{\left[s+\tau_{3}, \infty\right)}$. This shows that $w_{1}$ is switched to 0 in $\mathfrak{B}$. Similarly we can prove that there exists $\tau_{4}>0, w_{4} \in \mathfrak{B}$ such that $\left.w_{4}\right|_{(-\infty, s]}=\left.0\right|_{(-\infty, s]}$ and $\left.w_{4}\right|_{\left[s+\tau_{4}, \infty\right)}=\left.w_{2}\right|_{\left[s+\tau_{4}, \infty\right)}$. Write $\tau_{5}=\max \left\{\tau_{3}, \tau_{4}\right\}, w_{5}=w_{3}+w_{4}$. Then we see that $w_{5} \in \mathfrak{B},\left.w_{5}\right|_{(-\infty, s]}=\left.w_{1}\right|_{(-\infty, s]}$ and $\left.w_{5}\right|_{\left[s+\tau_{5}, \infty\right)}=\left.w_{2}\right|_{\left[s+\tau_{5}, \infty\right)}$. This shows that $\mathfrak{B}_{2}$ is $\mathfrak{B}$-controllable. The maximality of $\mathfrak{B}_{\text {cont }}$ along with $\mathfrak{B}_{\text {cont }} \subset \mathfrak{B}_{2}$ implies that the two behaviours are the same. Hence $\mathfrak{B}_{\text {cont }}$ is unique.

In the rest of this paper, for a linear behaviour $\mathfrak{B}$, we always use $\mathfrak{B}_{\text {cont }}$ to denote its unique maximal $\mathfrak{B}$-controllable sub-behaviour.

The above proof also shows that the sum of any two linear $\mathfrak{B}$-controllable sub-behaviours is $\mathfrak{B}$-controllable and, therefore, so is the sum of all linear $\mathfrak{B}$-controllable sub-behaviours. Hence

$$
\mathfrak{B}_{\text {cont }}=\operatorname{span}\{B \subset \mathfrak{B}: B \text { is linear and } \mathfrak{B} \text {-controllable }\} .
$$

Next we show that shift invariance is preserved for the unique maximal $\mathfrak{B}$-controllable subbehaviours. In particular, in the shift invariant linear setting, $\mathfrak{B}_{\text {cont }}$ is linear and shift invariant.

Lemma 2.9 Suppose $\mathfrak{B}$ is shift invariant and has a unique maximal $\mathfrak{B}$-controllable sub-behaviour $\mathfrak{B}^{*}$. Then $\mathfrak{B}^{*}$ is shift invariant.

Proof. Let $r, s \in \mathcal{T}, \sigma_{r} w_{1}, \sigma_{r} w_{2} \in \sigma_{r} \mathfrak{B}^{*}$ with $w_{1}, w_{2} \in \mathfrak{B}^{*}$. Then there exist $w_{3} \in \mathfrak{B}$ and $\tau>0$ such that $w_{3}(t)=w_{1}(t)$ for $t \leq s+r$ and $w_{3}(t)=w_{2}(t)$ for $t \geq s+r+\tau$. Hence

$$
\sigma_{r} w_{3}(t)= \begin{cases}\sigma_{r} w_{1}(t), & \text { if } t \leq s \\ \sigma_{r} w_{2}(t), & \text { if } t \geq s+\tau\end{cases}
$$

Since $\sigma_{r} w_{3} \in \sigma_{r} \mathfrak{B}=\mathfrak{B}$, we see $\sigma_{r} \mathfrak{B}^{*}$ is $\mathfrak{B}$-controllable and hence $\sigma_{r} \mathfrak{B}^{*} \subset \mathfrak{B}^{*}$ as $\mathfrak{B}^{*}$ is the unique maximal $\mathfrak{B}$-controllable sub-behaviour.

Corollary 2.10 Suppose $\mathfrak{B}$ is linear and shift invariant. Then, $\mathfrak{B}_{\text {cont }}$ is linear and shift invariant.

We conclude this section by showing that $\mathfrak{B}$-controllable linear sub-behaviours inherit memory properties from the original behaviour $\mathfrak{B}$ : 
Lemma 2.11 Let $\mathfrak{B}$ be a linear shift invariant behaviour with finite memory $l \geq 0$. Then $\mathfrak{B}_{\text {cont }}$ has memory $l \geq 0$.

Proof. First of all, we need a new notion: a sub-behaviour $\mathfrak{B}^{*}$ is $0-\mathfrak{B}$-controllable if it is shift invariant and given any $w_{1}, w_{2} \in \mathfrak{B}^{*}$, there exist $w_{3} \in \mathfrak{B}$ and $\tau \in \mathcal{T}_{+}$satisfying equation (2.7). Using the same procedure as used in Theorem 2.8, we can see that a maximal linear 0 - $\mathfrak{B}$-controllable sub-behaviour of $\mathfrak{B}$ exists, denoted by $\mathfrak{B}_{\text {cont }}^{0}$, which is is shift invariant. By the remark following Definition 2.5, $\mathfrak{B}_{\text {cont }}=\mathfrak{B}_{\text {cont }}^{0}$.

Now, let $w_{1}, w_{2} \in \mathfrak{B}_{\text {cont }}$ with $\left.w_{1}\right|_{[0, l]}=\left.w_{2}\right|_{[0, l]}$. Then

$$
w_{3}(t)=\left\{\begin{array}{l}
w_{1}(t), \text { if } t<0 \\
w_{2}(t), \text { if } t \geq 0
\end{array} \in \mathfrak{B} .\right.
$$

Since $\mathfrak{B}_{\text {cont }}$ is $\mathfrak{B}$-controllable, for any $w \in \mathfrak{B}_{\text {cont }}$, there exist $\tau_{1}>0$ and $v_{1} \in \mathfrak{B}$ such that $\left.v_{1}\right|_{(-\infty, 0]}=\left.w_{1}\right|_{(-\infty, 0]}=\left.w_{3}\right|_{(-\infty, 0]}$ and $\left.v_{1}\right|_{\left[\tau_{1}, \infty\right)}=\left.w\right|_{\left[\tau_{1}, \infty\right)}$, that is, $w_{3}$ can be switched to $w$. Similarly, there exist $\tau_{2}>0$ and $v_{2} \in \mathfrak{B}$ such that $\left.v_{2}\right|_{(-\infty, 0]}=\left.w\right|_{(-\infty, 0]}$ and $\left.v_{2}\right|_{\left[\tau_{2}, \infty\right)}=\left.w_{2}\right|_{\left[\tau_{2}, \infty\right)}=$ $\left.w_{3}\right|_{\left[\tau_{2}, \infty\right)}$, that is, $w$ can be switched to $w_{3}$. Hence $\operatorname{span}\left\{w_{3}\right\}+\mathfrak{B}_{\text {cont }}^{0}$ is 0 - $\mathfrak{B}$-controllable. By its maximality $w_{3} \in \mathfrak{B}_{\text {cont }}^{0}$ and therefore $w_{3} \in \mathfrak{B}_{\text {cont }}$ as shown above. This completes the proof.

We remark that all results in this section remain valid if the $\mathfrak{B}$-controllability is replaced by the controllability defined in Definition 2.4.

Given a linear differential behaviour $\mathfrak{B}$, a unique maximal controllable (as per Definition 2.4) sub-behaviour exists, denoted by $\mathfrak{B}_{c}$. Since this controllable sub-behaviour is $\mathfrak{B}$-controllable, $\mathfrak{B}_{c} \subset \mathfrak{B}_{\text {cont }}$. The next lemma shows that for certain behaviours (that can be represented as kernels of certain classes of operator), the two notions of controllability (definitions 2.4 and 2.5) coincide. In particular, for differential/difference behaviours, $\mathfrak{B}_{\text {cont }}$ equals $\mathfrak{B}_{c}$.

We introduce the following notation. Let $R: \operatorname{dom}(R) \rightarrow \operatorname{im}(R) \subset \operatorname{map}\left(\mathcal{T}, \mathbb{R}^{n_{2}}\right)$ be an operator, where $\operatorname{dom}(R) \subset \operatorname{map}\left(\mathcal{T}, \mathbb{R}^{n_{1}}\right)$ is the domain of $R$ and $\operatorname{im}(R) \subset \operatorname{map}\left(\mathcal{T}, \mathbb{R}^{n_{2}}\right)$ denotes the image of $R$.

Definition 2.12 Let $R: \operatorname{dom}(R) \rightarrow \operatorname{im}(R) \subset \operatorname{map}\left(\mathcal{T}, \mathbb{R}^{n_{2}}\right)$ be an operator, where $\operatorname{dom}(R) \subset$ $\operatorname{map}\left(\mathcal{T}, \mathbb{R}^{n_{1}}\right) . R$ is said to have local action if there exists $k_{2} \geq k_{1}>0$ such that for all $t \in \mathcal{T}$, $w_{1}, w_{2} \in \operatorname{dom}(R)$ with $\left.w_{1}\right|_{\left[t-k_{2}, t+k_{2}\right]}=\left.w_{2}\right|_{\left[t-k_{2}, t+k_{2}\right]}$ implies $\left.\left(R w_{1}\right)\right|_{\left[t-k_{1}, t+k_{1}\right]}=\left.\left(R w_{2}\right)\right|_{\left[t-k_{1}, t+k_{1}\right]}$.

Differential operators, (backward) difference operators and delay-differential operators all have local action property. For differential operators, the constants $k_{1}, k_{2}$ can be as small as possible. For difference/delay operators, $k_{2}-k_{1}$ should be greater or equal to the maximum of differences/delays involved.

Lemma 2.13 Let $\mathfrak{B}$ be a linear behaviour and $\mathfrak{B}_{c}$ be the maximal controllable sub-behaviour of $\mathfrak{B}$ (as per Definition 2.4). Let $R: \operatorname{dom}(R) \rightarrow \operatorname{map}\left(\mathcal{T}, \mathbb{R}^{n_{1}}\right), R_{c}: \mathfrak{B} \rightarrow \operatorname{map}\left(\mathcal{T}, \mathbb{R}^{n_{2}}\right.$ ) and $S: \operatorname{im}\left(R_{c}\right) \rightarrow \operatorname{map}\left(\mathcal{T}, \mathbb{R}^{n_{1}}\right)$ be linear operators, where $\mathfrak{B} \subset \operatorname{dom}(R) \subset \operatorname{map}\left(\mathcal{T}, \mathbb{R}^{n}\right)$. Suppose:

(i) $R=S R_{c}$,

(ii) $\mathfrak{B}=\{w \in \operatorname{dom}(R), R w=0\}$ and $\mathfrak{B}_{c}=\left\{w \in \mathfrak{B}: R_{c} w=0\right\}$,

(iii) the behaviour $\mathfrak{B}_{S}=\left\{w \in \operatorname{im}\left(R_{c}\right): S w=0\right\}$ is autonomous, and

(iv) $R_{c}$ has local action.

Then $\mathfrak{B}_{\text {cont }}=\mathfrak{B}_{c}$. 
Proof. Let

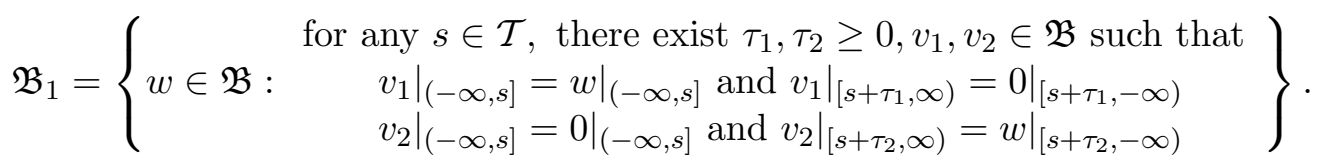

We claim $\mathfrak{B}_{\text {cont }}=\mathfrak{B}_{1}$ and $\mathfrak{B}_{1}=\mathfrak{B}_{c}$, so $\mathfrak{B}_{\text {cont }}=\mathfrak{B}_{c}$.

We first prove $\mathfrak{B}_{\text {cont }}=\mathfrak{B}_{1}$. Since $0 \in \mathfrak{B}_{\text {cont }}$, any $w \in \mathfrak{B}_{\text {cont }}$ and be patched to 0 in $\mathfrak{B}$ and vice versa. This shows $\mathfrak{B}_{\text {cont }} \subset \mathfrak{B}_{1}$. To show the reverse inclusion, let $w_{1}, w_{2} \in \mathfrak{B}_{1}$. Then for any $s \in \mathcal{T}$, there exists $\tau_{i} \geq 0$ and $u_{i}, v_{i} \in \mathfrak{B}(i=1,2)$ such that

$$
\begin{gathered}
\left.u_{i}\right|_{(-\infty, s]}=\left.w_{i}\right|_{(-\infty, s]},\left.\quad u_{i}\right|_{\left[s+\tau_{1}, \infty\right)}=\left.0\right|_{\left[s+\tau_{1},-\infty\right)}, \\
\left.v_{i}\right|_{(-\infty, s]}=\left.0\right|_{(-\infty, s]} \text { and }\left.v_{i}\right|_{\left[s+\tau_{2}, \infty\right)}=\left.w_{i}\right|_{\left[s+\tau_{2},-\infty\right)} .
\end{gathered}
$$

From this it follows that $u_{1}+v_{2}$ patches $w_{1}$ to $w_{2}$ and $u_{2}+v_{1}$ patches $w_{2}$ to $w_{1}$. So $\operatorname{span}\left\{w_{1}, w_{2}\right\}$ is $\mathfrak{B}$-controllable and therefore $w_{1}, w_{2} \in \mathfrak{B}_{\text {cont }}$ which proves $\mathfrak{B}_{1} \subset \mathfrak{B}_{\text {cont }}$.

We now prove $\mathfrak{B}_{1}=\mathfrak{B}_{c}$. Since $\mathfrak{B}_{c} \subset \mathfrak{B}_{\text {cont }}=\mathfrak{B}_{1}$, we only need to prove $\mathfrak{B}_{1} \subset \mathfrak{B}_{c}$. Suppose it is not the case. Then there exists $w \in \mathfrak{B}_{1} \backslash \mathfrak{B}_{c}$ such that $R_{c} w \neq 0$, that is, there exists $t_{0} \in \mathcal{T}$ with $R_{c} w\left(t_{0}\right) \neq 0$. Since $R_{c}$ has local action, there exist $k_{2}>k_{1}>0$ such that for all $t \in \mathcal{T}$,

$$
w_{1}, w_{2} \in \mathfrak{B} \text { with }\left.w_{1}\right|_{\left[t-k_{2}, t+k_{2}\right]}=\left.w_{2}\right|_{\left[t-k_{2}, t+k_{2}\right]} \text { implies }\left.\left(R_{c} w_{1}\right)\right|_{\left[t-k_{1}, t+k_{1}\right]}=\left.\left(R_{c} w_{2}\right)\right|_{\left[t-k_{1}, t+k_{1}\right]} .
$$

Let $s \in \mathcal{T}$ such that $s>t_{0}+k_{2}$. By the definition of $\mathfrak{B}_{1}$, there exist $\tau>0$ and $w_{s} \in \mathfrak{B}$ such that

$$
\left.w_{s}\right|_{(-\infty, s]}=\left.w\right|_{(-\infty, s]} \text { and }\left.w_{s}\right|_{[s+\tau, \infty)}=\left.0\right|_{[s+\tau, \infty)} .
$$

Let $v_{s}=R_{c} w_{s}$. Since $w_{s} \in \mathfrak{B}=\{w: R w=0\}$, by assumption (i), $S v_{s}=S R_{c} w_{s}=R w_{s}=$ 0 and therefore $v_{s} \in \mathfrak{B}_{S}$. Since $R_{c}$ has local action and $\left.w_{s}\right|_{[s+\tau, \infty)}=\left.0\right|_{[s+\tau, \infty)}$, there exist $a, b \geq s+\tau, a<b$ such that $\left.v_{s}\right|_{[a, b]}=\left.R_{c} w_{s}\right|_{[a, b]}=\left.R_{c} 0\right|_{[a, b]}=\left.0\right|_{[a, b]}$. By the autonomy of $\mathfrak{B}_{S}$ (choosing $a, b$ such that $b-a>$ minimum memory of $\mathfrak{B}_{S}$ ), $v_{s} \equiv 0$, i.e. $R_{c} w_{s}=0$. Since $\left.w_{s}\right|_{(-\infty, s]}=\left.w\right|_{(-\infty, s]}$ and $t_{0}<s-k_{2}$, we see $\left.w_{s}\right|_{\left[t_{0}-k_{2}, t_{0}+k_{2}\right]}=\left.w\right|_{\left(t_{0}-k_{2}, t_{0}+k_{2}\right]}$. Therefore, by the local action assumption on $R_{c},\left(R_{c} w\right)\left(t_{0}\right)=\left(R_{c} w_{s}\right)\left(t_{0}\right)=0$. This is a contradiction and completes the proof.

We may now apply this lemma to linear differential, delay-differential and difference behaviours, that is behaviours defined by system of differential/delay-differential/difference equations. As shown in $[5,7]$, those behaviours are kernels of linear operators governed by matrices of polynomials.

Theorem 2.14 For a differential/delay-differential/difference behaviour $\mathfrak{B}$, its maximal $\mathfrak{B}$ controllable sub-behaviour $\mathfrak{B}_{\text {cont }}$ is the same as its maximal controllable sub-behaviour $\mathfrak{B}_{c}$.

Proof. We first suppose that $\mathfrak{B}$ is a differential behaviour. Both $\mathfrak{B}$ and $\mathfrak{B}_{c}$ have the representations:

$$
\mathfrak{B}=\left\{w \in \mathfrak{C}^{\infty}: R\left(\frac{d}{d t}\right) w=0\right\}, \quad \mathfrak{B}_{c}=\left\{w \in \mathfrak{B}: R_{c}\left(\frac{d}{d t}\right) w=0\right\},
$$

where $R(\xi), R_{c}(\xi)$ are $m \times n$ matrices of polynomials of $\xi$. Since $\mathfrak{B}_{c} \subset \mathfrak{B}$, there exists a nonsingular polynomial matrix $S$ such that $R=S R_{c}$. Moreover, the kernel of $S$ is an autonomous behaviour. So all assumptions of Lemma 2.13 hold and $\mathfrak{B}_{\text {cont }}=\mathfrak{B}_{c}$.

If $\mathfrak{B}$ is a delay-differential behaviour, as shown in [5], the proof is almost the same except that the matrix operators are $R\left(\frac{d}{d t}, \sigma\right), R_{c}\left(\frac{d}{d t}, \sigma\right)$ with $\sigma$ the delay operation. Where no differentiation operators are present, we obtain the proof for difference behaviour case. 
Earlier, we have shown that

$$
\mathfrak{B}_{\text {cont }}=\Sigma\{B \subset \mathfrak{B}: B \text { is linear and } \mathfrak{B} \text {-controllable }\} .
$$

Since $\mathfrak{B}_{c}=\mathfrak{B}_{\text {cont }}$ for a differential/difference behaviour $\mathfrak{B}$, this gives us a direct set-theoretic construction of $\mathfrak{B}_{c}$. To the best knowledge of the authors, this direct set-theoretic construction of $\mathfrak{B}_{c}$ does not appear in the literature. Within the behavioural literature, the controllable subbehaviour is typically constructed algebraically given the equations governing the behaviour, and it is shown via the duality between the behaviour and the algebraic structure that the controllable sub-behaviour is the 'largest' such subset. It is noteworthy to observe that in some settings (e.g. both $1 \mathrm{D}$ and $\mathrm{nD}$ differential systems), the existence of the corresponding maximal algebraic object appears constructively, see [7], [10].

\section{The Autonomous-Controllable Decomposition}

For 1D (one dimensional) differential/difference behaviours $\mathfrak{B}$ it is well known that $\mathfrak{B}$ can be split into a direct sum of the controllable and an autonomous part:

$$
\mathfrak{B}=\mathfrak{B}_{c} \oplus \mathfrak{B}_{a}
$$

where $\mathfrak{B}_{c} \subset \mathfrak{B}$ is the maximal controllable sub-behaviour of $\mathfrak{B}$ as per Definition 2.4 and $\mathfrak{B}_{a}$ is an autonomous sub-behaviour. Because of Theorem 2.14, we in fact have:

$$
\mathfrak{B}=\mathfrak{B}_{\text {cont }} \oplus \mathfrak{B}_{a}
$$

This direct sum decomposition is a special feature which only holds for certain classes of systems (such as the differential case [7]). For example, in the $\mathrm{nD}$ differential/difference setting, it is known that this sum is not direct for $n>1$ (see [18]), and in the context of delay differential systems it is only known that the sum is direct for commensurate delays [5]. However since an additive decomposition is critical to what follows, we do not restrict attention to direct sums, but treat it as an important special case.

Therefore this section examines both direct and non-direct sum decompositions at the trajectory level and examines the relationship between autonomy of any direct summand to the $\mathfrak{B}$ controllable part and the corresponding lack of controllability of this part. We wish to show that, under certain conditions, $\mathfrak{B}=\mathfrak{B}_{\text {cont }} \oplus \mathfrak{B}^{*}$ or $\mathfrak{B}=\mathfrak{B}_{\text {cont }}+\mathfrak{B}^{*}$ implies that $\mathfrak{B}^{*}$ is autonomous. Behaviours with such decomposition will be studied later on for stability and robustness. We have already established a connection between $\mathfrak{B}_{\text {cont }}$ and $\mathfrak{B}_{c}$ (the maximal controllable sub-behaviour of $\mathfrak{B}$ as per Definition 2.4). Note that in this paper we are only considering behaviours defined over the time set $\mathcal{T}$ which is either $\mathbb{Z}$ or $\mathbb{R}$.

We begin the study of these decompositions by first considering linear, shift invariant, autonomous behaviours:

Lemma 3.1 Let $\mathfrak{B}$ be linear, shift invariant autonomous behaviour. Let $\mathfrak{B}_{\text {cont }}$ be the maximal linear $\mathfrak{B}$-controllable sub-behaviour of $\mathfrak{B}$. Then $\mathfrak{B}_{\text {cont }}=\{0\}$.

Proof. Firstly it is obvious that $0 \in \mathfrak{B}_{\text {cont }}$. Suppose there exists $w \neq 0, w \in \mathfrak{B}_{\text {cont }}$. By $\mathfrak{B}$ controllability, there exist trajectories $w_{1}, w_{2} \in \mathfrak{B}$ and $\tau_{1}, \tau_{2}>0$ s.t. $\left.w_{1}\right|_{(-\infty, 0]}=0,\left.w_{1}\right|_{\left(\tau_{1}, \infty\right)}=$ $\left.w\right|_{\left(\tau_{1}, \infty\right)}$ and $\left.w_{2}\right|_{(-\infty, 0]}=\left.w\right|_{(-\infty, 0]},\left.w_{2}\right|_{\left(\tau_{2}, \infty\right)}=0$. By shift invariance, $\sigma_{-\tau_{2}-l_{0}} w_{1} \in \mathfrak{B}$ where $l_{0}$ is the minimum finite memory. Since $\left.\sigma_{-\tau_{2}-l_{0}} w_{1}\right|_{\left[\tau_{2}, \tau_{2}+l_{0}\right]}=0=\left.w_{2}\right|_{\left[\tau_{2}, \tau_{2}+l_{0}\right]}$, it follows from the autonomous assumption that $\sigma_{-\tau_{2}-l_{0}} w_{1}(t)=w_{2}(t)$ for all $t \in \mathcal{T}$. This tells $w_{2}(t)=0$ for all $t \in \mathcal{T}$ and therefore $w_{1}=\sigma_{\tau_{2}+l_{0}} w_{2}=0$. So $w \equiv 0$ which is a contradiction. 
Note that the above lemma is only in one direction.

For any $V \subset \mathcal{T}$, let $P_{V}$, denote the natural projection (restriction) of signals defined on $\mathcal{T}$ to signals defined on $V$. As a shorthand we write $P_{+}$for $P_{\mathcal{T}_{+}}$and $P_{-}$for $P_{\mathcal{T} \backslash \mathcal{T}_{+}}$.

Lemma 3.2 1) For any two behaviours $\mathfrak{B}_{1}, \mathfrak{B}_{2}$, we have $P_{V}\left(\mathfrak{B}_{1}+\mathfrak{B}_{2}\right)=P_{V} \mathfrak{B}_{1}+P_{V} \mathfrak{B}_{2}$.

2) If $\mathfrak{B}$ is a linear, shift invariant behaviour with finite memory $l>0$ and $\mathfrak{B}=\mathfrak{B}_{\text {cont }} \oplus \mathfrak{B}^{*}$ then $P_{V} \mathfrak{B}=P_{V} \mathfrak{B}_{\text {cont }} \oplus P_{V} \mathfrak{B}^{*}$ for all intervals $V$ of length greater than $l$.

Proof. Claim 1) is rather obvious. To establish claim 2), we need only to prove the sum $P_{V} \mathfrak{B}_{\text {cont }} \oplus P_{V} \mathfrak{B}^{*}$ is direct. Let $w_{1} \in \mathfrak{B}^{*}, w_{2} \in \mathfrak{B}_{\text {cont }}$ such that $\left.w_{1}\right|_{V}=\left.w_{2}\right|_{V}$, i.e. $\left.w_{1}\right|_{V}=\left.w_{2}\right|_{V} \in$ $P_{V} \mathfrak{B}_{\text {cont }} \cap P_{V} \mathfrak{B}^{*}$. Consider any $w_{3} \in \mathfrak{B}_{\text {cont }}$. By the memory property $w_{1}$ can be patched to $w_{2}$ and conversely, and by $\mathfrak{B}$-controllability $w_{2}$ can be patched to $w_{3}$ and conversely. This tells that $w_{1}$ can be patched to $w_{3}$ in $\mathfrak{B}$ and conversely. Hence $\mathfrak{B}^{\prime}:=\mathfrak{B}_{\text {cont }}+\operatorname{span}\left(w_{1}\right)$ is $\mathfrak{B}$-controllable. Since $\mathfrak{B}_{\text {cont }}$ is the unique maximal $\mathfrak{B}$-controllable subbehavior of $\mathrm{B}$, it must contain $\mathfrak{B}^{\prime}$. Hence $w_{1} \in \mathfrak{B}_{\text {cont }}$. By the direct sum property it follows that $w_{1}=0$, hence $\left.w_{1}\right|_{V}=\left.w_{2}\right|_{V}=0$.

Proposition 3.3 Let $\mathfrak{B}$ be a linear, shift invariant behaviour with finite memory. Then the following are equivalent:

1. $\mathfrak{B}_{\text {cont }}$ splits $\mathfrak{B}$, i.e. $\mathfrak{B}=\mathfrak{B}_{\text {cont }} \oplus \mathfrak{B}^{*}$ for some subbehaviour $\mathfrak{B}^{*} \subset \mathfrak{B}$.

2. There exists a behaviour $\mathfrak{B}^{*}$ for which $\left(\mathfrak{B}^{*}\right)_{\text {cont }}=\{0\}$ (where $\left(\mathfrak{B}^{*}\right)_{\text {cont }}$ is the maximal linear $\mathfrak{B}$-controllable sub-behaviour of $\left.\mathfrak{B}^{*}\right)$ and $\mathfrak{B}=\mathfrak{B}_{\text {cont }} \oplus \mathfrak{B}^{*}$.

Proof. That 2. implies 1 . is obvious. It remains to show 1 . implies 2 . Since $\mathfrak{B}_{\text {cont }}$ splits $\mathfrak{B}$, there exists $\mathfrak{B}^{*}$ such that $\mathfrak{B}=\mathfrak{B}_{\text {cont }} \oplus \mathfrak{B}^{*}$. Then $\left(\mathfrak{B}^{*}\right)_{\text {cont }} \subset \mathfrak{B}_{\text {cont }} \cap \mathfrak{B}^{*}=\{0\}$.

Note that if one considers the maximal $\mathfrak{B}^{*}$-controllable sub-behaviour of $\mathfrak{B}^{*}$, instead of $\left(\mathfrak{B}^{*}\right)$ cont, then the conclusion of Proposition 3.3 continues to hold. At this juncture, we would like to comment that since Lemma 3.1 is only in one direction, we cannot conclude that $\mathfrak{B}^{*}$ in Proposition 3.3 is autonomous. We further observe that if $\mathfrak{B}_{c}$ (the controllable part of a behaviour $\mathfrak{B}$ as per Definition 2.4) has finite co-dimension (as in the differential ([8]) and commensurate delay ([5]) settings), then it is known that $\mathfrak{B}_{c}$ splits $\mathfrak{B}$. In these cases, we know that the summand $\mathfrak{B}^{*}$ is autonomous (by the traditional definition)

Unfortunately the autonomy of $\mathfrak{B}^{*}$ in the present situation remains a problem. So we introduce the following definition for the rest of this paper.

Definition 3.4 A behaviour $\mathfrak{B}$ is said to have a controllable-autonomous decomposition if there exists a autonomous sub-behaviour of $\mathfrak{B}$, denoted by $\mathfrak{B}_{\text {aut }}$, such that

$$
\mathfrak{B}=\mathfrak{B}_{\text {cont }}+\mathfrak{B}_{\text {aut }} .
$$

\section{Stability}

Stability is determined by the signal spaces involved. We will consider the spaces $L^{p}\left(\mathcal{T}, \mathbb{R}^{n}\right)$ with $0 \leq p \leq \infty$. In the case when $\mathcal{T}=\mathbb{R}$, it is the standard $L^{p}$ spaces such as $L^{2}\left(\mathbb{R}, \mathbb{R}^{n}\right)$ and $L^{\infty}\left(\mathbb{R}, \mathbb{R}^{n}\right)$ for continuous-time signals. In the case when $\mathcal{T}=\mathbb{Z}$, it becomes the standard $l^{p}$ spaces used for discrete signals. 
Given a general normed signal space (say) $Y$ of signals from $\mathcal{T}$ or $\mathcal{T}_{+}$to $\mathbb{R}^{n}$, the corresponding extended space $Y_{e}$ is defined as:

$$
Y_{e}=\left\{y: \mathcal{I} \rightarrow \mathbb{R}^{n}: T_{\tau} y \in Y \text { for all } \tau \in \mathcal{I}_{+}\right\}
$$

where $\mathcal{I}=\mathcal{T}$ or $\mathcal{T}_{+}$subject to on which set the space $Y$ is defined, and $T_{\tau}$ is the truncation operator, that is $\left(T_{\tau} y\right)(t)=y(t)$ for $t \leq \tau$ and 0 for $t>\tau$.

Since this section, the behaviours considered will be restricted to be within the extended signal spaces $L_{e}^{p}:=L_{e}^{p}\left(\mathcal{T}, \mathbb{R}^{n}\right), 1 \leq p \leq \infty$, i.e., $L^{p}$ behaviours or subsets of $L_{e}^{p}$.

As a shorthand we denote by $X=L^{p}\left(\mathcal{T}_{+}\right)=: L^{p}\left(\mathcal{T}_{+}, \mathbb{R}^{n}\right), 1 \leq p \leq \infty$. So $X_{e}=L_{e}^{p}\left(\mathcal{T}_{+}\right)$. We remark that when the results do not need a normed structure on the signal spaces, our discussions and definitions also remain valid for $C^{\infty}$ behaviours (with $X=C^{\infty}\left(\mathcal{T}_{+}\right)$).

We generalise the standard behavioural definition of stability for autonomous systems as follows:

Definition 4.1 An autonomous system $\mathfrak{B}_{\text {aut }}$ is said to be $X$-stable if for any $w \in \mathfrak{B}_{\text {aut }}$, $\left.w\right|_{[0, \infty)} \in X$.

This notion of stability can be equivalently expressed as the statement that $\mathfrak{B}_{\text {aut }}$ is stable if and only if $P_{+} \mathfrak{B}_{\text {aut }} \subset X$. For non-autonomous systems, we adopt the following stability concept for behaviours with i/o partition (see [7]), which captures the notion of 'whatever the past, given a bounded future input, the future output is bounded':

Definition 4.2 A behaviour $\mathfrak{B}$ with i/o partition $u \mid y$ is $X$-stable if for all $(u, y) \in \mathfrak{B}$ with $\left.u\right|_{\mathcal{T}_{+}} \in X$ we have $\left.y\right|_{\mathcal{T}_{+}} \subset X$.

When $X$ is given, throughout the paper we refer to the notion of ' $X$-stability' simply as 'stability'. Associated to any behaviour are the stable sub-behaviours which correspond to the behaviour taking zero values up to time $t=0$ :

Definition 4.3 The graph $\mathcal{G}_{\mathfrak{B}}$ of a behaviour $\mathfrak{B}$ is defined to be:

$$
\mathcal{G}_{\mathfrak{B}}:=\left\{w \in X \mid \text { there exists } v \in \mathfrak{B} \text { such that }\left.v\right|_{\mathcal{T}_{-}}=0,\left.v\right|_{\mathcal{T}_{+}}=\left.w\right|_{\mathcal{T}_{+}}\right\} .
$$

The extended graph $\mathcal{Z}_{\mathfrak{B}}$ of $\mathfrak{B}$ is defined to be

$$
\mathcal{Z}_{\mathfrak{B}}:=\left\{w \in X_{e} \mid \text { there exists } v \in \mathfrak{B} \text { such that }\left.v\right|_{\mathcal{T}_{-}}=0,\left.v\right|_{\mathcal{T}_{+}}=\left.w\right|_{\mathcal{T}_{+}}\right\} .
$$

Note that when $\mathcal{T}=\mathbb{R}, X=L^{2}\left(\mathbb{R}_{+}\right), \mathcal{G}_{\mathfrak{B}}$ corresponds to the classical $\mathcal{H}^{2}$ graph ([13]).

Lemma 4.4 Let $\mathfrak{B}=\mathfrak{B}_{\text {cont }} \oplus \mathfrak{B}_{\text {aut }}$ be a linear, shift invariant behaviour with finite memory. Then $\mathcal{Z}_{\mathfrak{B}_{\text {cont }}}=\mathcal{Z}_{\mathfrak{B}}$ and $\mathcal{G}_{\mathfrak{B}_{\text {cont }}}=\mathcal{G}_{\mathfrak{B}}$.

Proof. $\quad$ Since $\mathfrak{B}_{\text {cont }} \subset \mathfrak{B}$ it follows that $\mathcal{Z}_{\mathfrak{B}_{\text {cont }}} \subset \mathcal{Z}_{\mathfrak{B}}$ and $\mathcal{G}_{\mathfrak{B}_{\text {cont }}} \subset \mathcal{G}_{\mathfrak{B}}$. Conversely, let $w \in \mathcal{Z}_{\mathfrak{B}}$. By the direct sum, there exists $w_{1} \in \mathfrak{B}_{\text {cont }}, w_{2} \in \mathfrak{B}_{\text {aut }}$ such that $w=w_{1}+w_{2}$. By definition of $\mathcal{Z}_{\mathfrak{B}}$, it follows that $\left.\left(w_{1}+w_{2}\right)\right|_{\mathcal{T}_{-}}=0$, so by Lemma 3.2 (ii), $\left.w_{1}\right|_{\mathcal{I}_{-}}=\left.w_{2}\right|_{\mathcal{T}_{-}}=0$. By the autonomy of $\mathfrak{B}_{\text {aut }}$, it follows that $w_{2}=0$. Hence $\mathcal{Z}_{\mathfrak{B}} \subset \mathcal{Z}_{\mathfrak{B}_{\text {cont }}}$ and $\mathcal{G}_{\mathfrak{B}} \subset \mathcal{G}_{\mathfrak{B}_{\text {cont }}}$.

We now introduce a notion of uniform stability, which captures the property that in addition to stability, there is a uniform gain between future inputs and outputs when the past is zero. We will discuss the relation between this notion of stability and dissipativity descriptions of stability in Section 8. 
Definition 4.5 A linear behaviour with i/o partition u|y is uniformly stable if

1. $\mathfrak{B}$ is stable.

2. There exists a bounded operator $\Psi: X \rightarrow X$ such that for all $(u, y) \in \mathfrak{B}$ such that $\left.u\right|_{\mathcal{T}_{+}} \in X$, $\left.(u, y)\right|_{\mathcal{T}_{-}}=0$ it follows that $y=\Psi(u)$.

Note that the existence of a single stable autonomous sub-behaviour $\mathfrak{B}_{\text {aut }}$ s.t. $\mathfrak{B}=\mathfrak{B}_{\text {cont }} \oplus \mathfrak{B}_{\text {aut }}$ does not imply stability. For an example, consider $\dot{x}=x+u, \dot{z}=-z, y=x+z$. Then the sub-behaviour generated by $\dot{z}=-z, u=x=0, y=z$ is stable and has the direct sum property and yet the behaviour is not stable. However, in the context of differential systems, this property characterizes stabilizability (see [7] for the case of $X=C_{0}\left(\mathbb{R}_{+}\right)$:

Definition 4.6 A behaviour $\mathfrak{B}$ is said to be stabilizable if for all $w_{1} \in \mathfrak{B}$, there exists $w_{2} \in \mathfrak{B}$ s.t. $\left.w_{1}\right|_{(-\infty, 0]}=\left.w_{2}\right|_{(-\infty, 0]}$ and $\left.w_{2}\right|_{[0, \infty)} \in X$.

A useful sufficient condition for stabilizability is as follows:

Lemma 4.7 Let $\mathfrak{B}$ be a linear shift invariant behaviour with finite memory. If $\mathfrak{B}=\mathfrak{B}_{\text {cont }}+\mathfrak{B}_{\text {aut }}$ and $\mathfrak{B}_{\text {aut }}$ is stable, then $\mathfrak{B}$ is stabilizable.

Proof. Suppose there exists a stable autonomous sub-behaviour $\mathfrak{B}_{\text {aut }}$ s.t. $\mathfrak{B}=\mathfrak{B}_{\text {cont }}+\mathfrak{B}_{\text {aut }}$. Then given any $w \in \mathfrak{B}$, there exist $w_{1} \in \mathfrak{B}_{\text {cont }}, w_{2} \in \mathfrak{B}_{\text {aut }}$ s.t. $w=w_{1}+w_{2}$. By controllability and shift invariance of $\mathfrak{B}_{\text {cont }}, w_{1}$ can be patched with $0 \in \mathfrak{B}_{\text {cont }}$ so there exists $w_{1}^{\prime} \in X$ s.t. $\left.w_{1}\right|_{(-\infty, 0]}=\left.w_{1}^{\prime}\right|_{(-\infty, 0]}$. By the stability of $\mathfrak{B}_{\text {aut }}, w_{2} \in X$. Hence $w^{\prime}=w_{1}^{\prime}+w_{2} \in X$ and $\left.w\right|_{(-\infty, 0]}=\left.w^{\prime}\right|_{(-\infty, 0]}$, thus $\mathfrak{B}$ is stabilizable as required.

It is natural to ask whether the converse holds, that is, whether stabilizability of $\mathfrak{B}$ implies stability of $\mathfrak{B}_{\text {aut }}$. For differential systems, Theorem 5.2.30 in [7] establishes the equivalence. For delay-differential systems with commensurate delays, the equivalence has been conjectured in [5], (p.117). It is thus useful to define the weaker notion of 'soundly stabilizable', and formally to note the equivalence of 'soundly stabilizable' and 'stabilizable' for differential systems as follows:

Definition $4.8 A$ behaviour $\mathfrak{B}$ is said to be soundly stabilizable if there exists a stable $\mathfrak{B}_{\text {aut }}$ such that $\mathfrak{B}=\mathfrak{B}_{\text {aut }}+\mathfrak{B}_{\text {cont }}$.

Proposition 4.9 For a differential behaviour $\mathfrak{B}, \mathfrak{B}$ is stabilizable if and only if it is soundly stabilizable.

Proof. See Theorem 5.2.30 in [7].

\section{Interconnections}

We are primarily interested in the standard feedback interconnections shown in Figures 1 and 2 .

Definition 5.1 Given a plant behaviour $\mathfrak{B}^{P}$, a controller behaviour $\mathfrak{B}^{C}$ and interconnection behaviour $\mathfrak{B}^{I}$ :

$$
\mathfrak{B}^{I}=\left\{\left(w_{0}, w_{1}, w_{2}\right)^{T} \in X_{e} \mid w_{0}=w_{1}+w_{2}\right\}
$$

we define the closed loop behaviour $\mathfrak{B}^{P \wedge{ }_{I} C}$ as follows:

$$
\mathfrak{B}^{P \wedge_{I} C}=\left\{\left(w_{0}, w_{1}, w_{2}\right)^{T} \in \mathfrak{B}^{I} \mid w_{1} \in \mathfrak{B}^{P}, w_{2} \in \mathfrak{B}^{C}\right\} .
$$




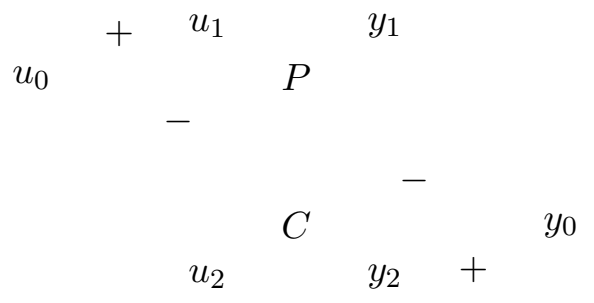

Figure 1: The closed-loop.

$\mathfrak{B}^{P}$
$w_{1}$
$w_{2}$
$\mathfrak{B}_{0}$

Figure 2: The interconnected behaviours: $w_{i}=\left(u_{i}, y_{i}\right)^{T}, i=0,1,2$.

To ensure uniqueness of solutions of the closed loop (modulo the autonomous part of the behaviour) we adopt the following definition:

Definition 5.2 Given a plant behaviour $\mathfrak{B}^{P}$, a controller behaviour $\mathfrak{B}^{C}$ and interconnection behaviour $\mathfrak{B}^{I}$ (5.12), the behaviour $\mathfrak{B}^{P \wedge_{I} C}$ is said to be well posed if

$$
X_{e}=\mathcal{Z}_{\mathfrak{B}^{P}} \oplus \mathcal{Z}_{\mathfrak{B}^{C}}
$$

This captures the idea that for the interconnection of behaviours with zero past, ' $w_{0}$ is an input, and for any input $w_{0}$, there exist unique internal signals $w_{1}, w_{2}$ '.

By (5.13), any $w_{0} \in X_{e}$ has a unique decomposition $w_{0}=w_{1}+w_{2}$ with $w_{1} \in \mathcal{Z}_{\mathfrak{B}^{P}}$ and $w_{2} \in \mathcal{Z}_{\mathfrak{B}^{C}}$. Hence two projection operators can be defined as below:

$$
\begin{array}{ll}
\Pi_{P / / C}: X_{e} \rightarrow \mathcal{Z}_{\mathfrak{B}^{P}}, & w_{0} \mapsto w_{1}, \\
\Pi_{C / / P}: X_{e} \rightarrow \mathcal{Z}_{\mathfrak{B}^{C}}, & w_{0} \mapsto w_{2} .
\end{array}
$$

Due to the interconnection behaviour, we have

$$
\Pi_{P / / C}+\Pi_{C / / P}=I .
$$

Definition 5.3 A controller behaviour $\mathfrak{B}^{C}$ is said to be a stabilizing controller for a plant behaviour $\mathfrak{B}^{P}$ if $\mathfrak{B}^{P \wedge_{I} C}$ is stable.

\section{Double Axis Time Theories}

In [3] it is shown that classical notions of stability and causality lead to problematic inconsistencies when input-output systems defined over a doubly infinite time axis are considered. In particular a causal and stable system $P_{h}$ was considered, defined by the convolution:

$$
P_{h}: u \mapsto y \quad: \quad y(t)=\int_{-\infty}^{\infty} h(t-\tau) u(\tau) d \tau=(h * u)(t), \quad h= \begin{cases}\exp (t) & \text { if } t \geq 0, \\ 0 & \text { otherwise }\end{cases}
$$


The interconnection of $P_{h}$ with a controller $C$ implementing negative unity feedback with gain greater than one as in Figure 1 was considered. It was shown [3] that if such a closed loop is considered to be well posed and stable (in the sense that the (single valued) map $\Pi: L^{2}\left(\mathbb{R}_{+}\right) \rightarrow$ $L^{2}\left(\mathbb{R}_{+}\right)$defined by $\left(\begin{array}{c}u_{0} \\ y_{0}\end{array}\right) \mapsto\left(\begin{array}{l}u_{1} \\ y_{1}\end{array}\right)$ exists (and necessarily has finite induced norm)), then necessarily the classical $L^{2}(\mathbb{R})$ graph of $P_{h}$ is closed. It was further shown in [3] that the trajectories

$$
\tilde{u}(t)=\left\{\begin{array}{ll}
\exp (-t) & t \geq 0 \\
0 & t<0
\end{array}, \quad \tilde{y}(t)=\left\{\begin{array}{ll}
-\frac{1}{2} \exp (-t) & t \geq 0 \\
-\frac{1}{2} \exp (t) & t<0
\end{array},\right.\right.
$$

can be obtained as the limit of a sequence of trajectories lying in the $L^{2}(\mathbb{R})$ graph of $P_{h}$, which is a contradiction since this solution does not satisfy the relation (6.16). Furthermore it can be shown [3] that if $w_{0}=(\tilde{u}, \tilde{y})^{T}$ acts as the disturbance to the closed loop $\left[P_{h}, C\right]$, where $C$ is negative unity feedback in Figure 1, then there is no solution $w_{1}=\left(u_{1}, y_{1}\right)^{T}$.

We view the above observations as indicating an inadequacy of (6.16) as a complete physical model. By linearity the input-output model (6.16) enforces $u=0$ implies $y=0$, hence there is no non-trivial autonomous sub-behaviour. By the natural inclusion of such autonomous subbehaviours, and with the corresponding relaxations of the notions of causality, well-posedness and stability, the example can be reconsidered as follows.

Let $\mathfrak{B}_{P_{h}}$ denote the smallest differential behaviour containing all trajectories $(u, y)$ satisfying (6.16). It can be observed that $\mathfrak{B}_{P_{h}}$ can be expressed by the following (minimal) kernel representation:

$$
\mathfrak{B}_{P_{h}}=\left\{w \in L_{\mathrm{loc}}^{1}(\mathbb{R}) \mid[-1 s-1] w=0\right\},
$$

where $s=\frac{d}{d t}$, and where solutions are interpreted in the weak sense. The behaviour $\mathfrak{B}_{P_{h}}$ can be explicitly expressed as:

$$
\mathfrak{B}_{P_{h}}=\left\{\left(\begin{array}{c}
u \\
y
\end{array}\right) \in L_{\mathrm{loc}}^{1}(\mathbb{R}) \mid y(t)=y_{0} \exp (t)+\int_{-\infty}^{t} \exp (t-\tau) u(\tau) d \tau, y_{0} \in \mathbb{R}, u \in L_{\mathrm{loc}}^{1}(\mathbb{R})\right\} .
$$

In terms of the definitions given in this paper, this behaviour is indeed stabilizable, and negative unity feedback with a gain greater than one provides a well-posed stabilizing interconnection: if $\mathfrak{B}_{C}=\left\{w \in L_{e}^{2}\left(\mathbb{R}_{+}\right) \mid w=(u, y)^{T}, u(t)=-k y(t)\right\}$ then $B^{P_{h} \wedge_{I} C}$ is (uniformly) stable for $k>1$. It is important to observe that

$$
\left(\begin{array}{c}
\tilde{u} \\
\tilde{y}
\end{array}\right)=\left(\begin{array}{c}
\tilde{u} \\
\exp (t)+\int_{-\infty}^{t} \exp (t-\tau) \tilde{u}(\tau) d \tau
\end{array}\right) \in B^{P_{h}} \cap L^{2}(\mathbb{R})
$$

since $(\tilde{u}, \tilde{y})^{T}$ can be explained by the sum of the forced solution $\left(\tilde{u}, \int_{-\infty}^{t} \exp (t-\tau) \tilde{u}(\tau) d \tau\right)^{T}$ and the unforced solution $(0, \exp (\cdot))^{T}$.

It is important to observe that the approach developed in this paper provides an alternative approach to addressing the classical problems of doubly infinite time axis. The approach taken here is perhaps half way between a double and a half line time axis, in that signals are defined over the whole of $\mathbb{R}$, but stability notions are related boundedness of signals when restricted to $\mathbb{R}_{+}$. Only in the case of uniform stability do we consider an induced norm and a zero past. The notion of well-posedness again restricts attention to the sub-behaviour with a zero past, and importantly does not impose uniqueness of solutions: $\left(w_{0}, w_{1}, w_{2}\right),\left(w_{0}, v_{1}, v_{2}\right) \in \mathfrak{B}^{P_{h} \wedge C}$ does not imply $\left(w_{1}, w_{2}\right)=\left(v_{1}, v_{2}\right)$ in general.

The approach considered in [9] identifies the operator $P_{h}$ defined by $(6.16)$ with its $L^{2}(\mathbb{R})$ closure $\bar{P}_{h}$. In this case, the closure exists and is the (stable) anti-causal operator $\bar{P}_{h}=P_{g}: L^{2}(\mathbb{R}) \rightarrow$ 
$L^{2}(\mathbb{R})$, where

$$
P_{g}: u \mapsto y \quad: \quad y(t)=\int_{-\infty}^{\infty} g(t-\tau) u(\tau) d \tau=(g * u)(t), \quad g= \begin{cases}-\exp (t) & \text { if } t \leq 0 \\ 0 & \text { otherwise }\end{cases}
$$

where $g(t)=-\exp (t)$ for $t \leq 0$ and zero otherwise. In [3], the identification of these two input-output systems is interpreted as 'more or less amount[ing] to abandoning any notion of causality', and it was stated that 'this is not a natural option, however, if the direction of time is well-defined'. It has the additional problem that $\bar{P}_{h}$ is stable, whose response on the bounded input $u(t)=1$ if $0 \leq t \leq 1, u(t)=0$ otherwise is the bounded output signal:

$$
y(t)= \begin{cases}0 & \text { if } t>1 \\ 1-\exp (1-t), & \text { if } 0 \leq t \leq 1 \\ \exp (t)-\exp (1+t), & \text { if } t<0\end{cases}
$$

We view this as problematic, since $P_{h}$ itself is defined as an operator $L^{2}(\mathbb{R}) \rightarrow L_{\text {loc }}^{2}(\mathbb{R})$, with the following unbounded output response to the above input:

$$
y(t)= \begin{cases}\exp (t)-\exp (1+t) & \text { if } t>1 \\ 1-\exp (t), & \text { if } 0 \leq t \leq 1 \\ 0, & \text { if } t<0\end{cases}
$$

These problems are only avoided in the case whereby the input-output operator has causal closure. In the discrete setting, the class of such transfer functions has been precisely characterised in [6] as the class of all Smirnoff functions; a class which includes all causal stable operators and excludes all causal unstable operators (as in the example considered); thus indicating that intrinsic difficulties with the input-output theory over $\mathbb{R}$.

In common with these other approaches to resolving the so-called 'Georgiou-Smith' paradox, the procedure of identifying the convolution system (6.16) with the smallest differential behaviour containing the same input-output pairs also identifies the same behaviour $\mathcal{B}_{P}=\mathcal{B}_{P_{h}}=\mathcal{B}_{P_{g}}$ to the anti-causal input-output system (6.18) as it is easily verified that $\mathfrak{B}_{P}$ contains all trajectories $(u, y) \in L_{\text {loc }}^{1}$ satisfying $(6.18)^{1}$. However, the consideration of $\mathcal{B}_{P}$ permits us to maintain a sensible notion of causality as follows:

Definition 6.1 A behaviour $\mathfrak{B}$ with input-output partition $(u, y)$ is said to be causal if

$$
T_{\tau} u_{1}=T_{\tau} u_{2} \quad \Longrightarrow \quad T_{\tau} \mathfrak{B}_{u_{1}}=T_{\tau} \mathfrak{B}_{u_{2}}
$$

where $\mathfrak{B}_{u}=\{w \in \mathfrak{B} \mid \exists y$ s.t. $w=(u, y) \in \mathfrak{B}\}$.

This can be interpreted as stating that the set of all past trajectories which can be generated from a particular past input cannot be affected by changing the future input, and represents a generalisation of the notion of a causal operator (where the non-uniqueness of the output given the input is suitably accounted for). We can now observe that $\mathfrak{B}_{P}$ indeed preserves causality, and we have thus arrived at a position whereby we can consider a suitable treatment of the system (6.16) in which the physical object under study can be thought of as causal and stabilizable. We find the explanation of trajectories of the system as a combination of an autonomous unforced sub-behaviour and a causal input to be more in line with physical thinking than the interpretation of the trajectories arising from a non-causal input to a single valued operator.

\footnotetext{
${ }^{1}$ Importantly however, $\mathcal{B}_{P}$ also includes unbounded trajectories such as $(0, \exp (\cdot))^{T}$ which are neither of the form $\left(u, P_{h} u\right)^{T}$ nor $\left(u, P_{g} u\right)$.
} 


\section{$7 \quad$ A Behavioural Generalisation of the Gap Metric and Robust Stability}

In this section we will be concerned with deriving the behavioural version of the central robust stability theorem for LTI systems. Our concern, for now, is with behaviours whose underlying signal space is equipped with a norm $\|\cdot\|$, that is $X$ is a vector space and all behaviours $\mathfrak{B}$ are such that $P_{+} \mathfrak{B} \subset X_{e}$. Furthermore, we assume that $(X,\|\cdot\|)$ has the property that $\left\|T_{\tau} x\right\| \leq a$ with $a>0$ for all $\tau \geq 0$ implies $x \in X$. The classical Lebesgue and Sobolev signal spaces, e.g. $X=L^{p}, l^{p}, W^{p, m} 1 \leq m, p \leq \infty$ satisfy this condition.

Definition 7.1 A mapping $\Psi: \operatorname{dom}(\Psi) \subset X_{e} \rightarrow X_{e}$ is said to be causal if $T_{\tau} \Psi w=T_{\tau} \Psi T_{\tau} w$ for all $w \in \operatorname{dom}(\Psi)$ and $\tau>0$ and with $T_{\tau} w \in \operatorname{dom}(\Psi)$. Its induced norm, denoted by $\|\Psi\|$, is defined as

$$
\|\Psi\|=\sup \left\{\frac{\left\|T_{\tau} \Psi w\right\|}{\left\|T_{\tau} w\right\|}: w \in \operatorname{dom}(\Psi), \tau>0, T_{\tau} w \neq 0 \text { and } T_{\tau} w \in \operatorname{dom}(\Psi)\right\} .
$$

Observe that $\left\|\Pi_{P / / C}\right\| \geq 1$ since for any $w_{0} \in \mathcal{G}_{\mathfrak{B}_{P}}, \Pi_{P / / C} w_{0}=w_{0}$. Motivated by [4] and the considerations in Section 4 we define the following notion of a gap distance between behaviours.

Definition 7.2 Given two behaviours $\mathfrak{B}^{1}, \mathfrak{B}^{2}$ define a gap functional:

$$
\begin{aligned}
\vec{\delta}\left(\mathfrak{B}^{1}, \mathfrak{B}^{2}\right) & = \begin{cases}\inf _{\Phi \in \mathcal{O}} \|\left.(I-\Phi)\right|_{\mathcal{G}_{\mathfrak{B}} 1} & \text { if } \mathfrak{B}^{2} \text { if soundly stabilizable } \\
1 & \text { if not, }\end{cases} \\
\delta\left(\mathfrak{B}^{1}, \mathfrak{B}^{2}\right) & =\max \left\{\vec{\delta}\left(\mathfrak{B}^{1}, \mathfrak{B}^{2}\right), \vec{\delta}\left(\mathfrak{B}^{2}, \mathfrak{B}^{1}\right)\right\}
\end{aligned}
$$

where

$$
\mathcal{O}=\left\{\Phi: \operatorname{dom}(\Phi) \subset \mathcal{G}_{\mathfrak{B}^{1}} \rightarrow \mathcal{G}_{\mathfrak{B}^{2}} \mid \Phi \text { bijective, causal, } \Phi(0)=0\right\}
$$

In the case of differential systems, the above definition of the gap can be related to the classical definitions as follows. Let $P_{1}(s), P_{2}(s)$ denote transfer functions corresponding to $\mathfrak{B}_{\text {cont }}^{P_{1}}, \mathfrak{B}_{\text {cont }}^{P_{2}}$ respectively, and let $\left(N_{i}, D_{i}\right) \in \mathcal{R} \mathcal{H}_{\infty}$ form normalized coprime factorizations of $P_{i}, i=1,2$. It follows that the classical graphs for $P_{1}(s)$ and $P_{2}(s)$ correspond (in the frequency domain) to the time domain graphs $\mathcal{G}_{\mathfrak{B}_{\text {cont }}^{1}}, \mathcal{G}_{\mathfrak{B}_{\text {cont }}^{2}}$. In the case where $X=L^{2}$, it has been shown in [4] and references therein that:

$$
\inf _{\Phi \in \mathcal{O}}\left\|\left.(I-\Phi)\right|_{\mathcal{G}_{P_{1}(s)}}\right\|=\vec{\delta}_{0}\left(P_{1}(s), P_{2}(s)\right)
$$

where the classical gap $\vec{\delta}_{0}$ can be expressed in a number of equivalent manners. Here we adopt an expression [13] which shows that the gap corresponds to the size of smallest stable co-prime factor perturbation between the two plants:

$$
\vec{\delta}_{0}\left(P_{1}(s), P_{2}(s)\right)=\inf \left\{\left\|\left(\begin{array}{c}
\Delta_{N} \\
\Delta_{D}
\end{array}\right)\right\|_{\mathcal{H}^{\infty}} \mid\left(\begin{array}{c}
\Delta_{N} \\
\Delta_{D}
\end{array}\right) \in \mathcal{R H}^{\infty}, P_{i}=\left(N_{i}+\Delta_{N}\right)\left(D_{i}+\Delta_{D}\right)^{-1}\right\} .
$$

In the context of differential systems, Lemma 4.4 shows that $\mathcal{G}_{\mathfrak{B}_{\text {cont }}^{1}}=\mathcal{G}_{\mathfrak{B}^{1}}, \mathcal{G}_{\mathfrak{B}_{\text {cont }}^{2}}=\mathcal{G}_{\mathfrak{B}^{2}}$ and Proposition 4.9 shows the equivalence between the concepts of sound stabilizability and stabilizability. Therefore it can be easily shown that the gap functional is determined as follows:

$$
\vec{\delta}\left(\mathfrak{B}^{1}, \mathfrak{B}^{2}\right)= \begin{cases}\vec{\delta}_{0}\left(P_{1}(s), P_{2}(s)\right) & \text { if } \mathfrak{B}^{2} \text { is stabilizable } \\ 1 & \text { if not, }\end{cases}
$$


We also remark, for completeness, that the directed gap can be computed via a standard $\mathcal{H}^{\infty}$ optimisation [2]:

$$
\vec{\delta}_{0}\left(P_{1}, P_{2}\right)=\inf _{Q \in \mathcal{H}_{\infty}}\left\|\left(\begin{array}{c}
D_{1} \\
N_{1}
\end{array}\right)-\left(\begin{array}{c}
D_{2} \\
N_{2}
\end{array}\right) Q\right\| .
$$

Observe that Definition 7.2 is a 'real' behavioural definition: everything is defined in terms of trajectories, and all sub-behaviours involved can be expressed in set-theoretic terms from the original behaviour $\mathfrak{B}$. From a behavioural perspective, it should also be noted that the definition does not require a distinguished i/o partition. It is natural to wish to substitute the condition of stabilizability for that of sound stabilizability in the definition of the gap functional, but as we have discussed previously the equivalence of these concepts is only known in the case of differential systems (see Proposition 4.9).

The central reason for consideration of gap distances in systems theory is to obtain robust stability results (see Theorem 7.6 later). In particular we want $\delta$ to capture the idea that any sensible stabilizing controller for $\mathfrak{B}^{P}$ will also stabilize $\mathfrak{B}^{P_{1}}$ provided $\delta\left(\mathfrak{B}^{P}, \mathfrak{B}^{P_{1}}\right)$ is small. By definition, the distance between $\mathfrak{B}$ and $\mathfrak{B}_{\text {cont }}$ is zero if $\mathfrak{B}$ is soundly stabilizable - this is reasonable since any stabilizing controller for $\mathfrak{B}$ will automatically stabilize $\mathfrak{B}_{\text {cont }}$ since $\mathfrak{B}_{\text {cont }} \subset$ $\mathfrak{B}$. Consequently $\delta$ is necessarily at most a pseudo-metric; indeed the distance between two stabilizable differential systems with the same transfer function will be 0 (since the graphs of the behaviours are identical, the minimizing $\Phi$ in the definition of the gap distance can be taken to be the identity, hence the gap distance is zero).

For the controllable-autonomous decomposition of the interconnected behaviour, we have:

Lemma 7.3 Suppose $\mathfrak{B}^{P}, B^{C}$ are linear behaviours with controllable-autonomous decompositions $\mathfrak{B}^{P}=\mathfrak{B}_{\text {cont }}^{P}+\mathfrak{B}_{\text {aut }}^{P}, \mathfrak{B}^{C}=\mathfrak{B}_{\text {cont }}^{C}+\mathfrak{B}_{\text {aut }}^{C}$. Then $\mathfrak{B}^{P{ }_{I} C}$ has the controllable-autonomous decomposition: $\mathfrak{B}^{P \wedge_{I} C}=\mathfrak{B}_{\text {cont }}^{P \wedge_{I} C}+\mathfrak{B}_{\text {aut }}^{P \wedge_{I} C}$ and

$$
\begin{aligned}
& \mathfrak{B}_{\text {cont }}^{P \wedge_{I} C}=\left\{\left(w_{1}+w_{2}, w_{1}, w_{2}\right) \mid w_{1} \in \mathfrak{B}_{\text {cont }}^{P}, w_{2} \in \mathfrak{B}_{\text {cont }}^{C}\right\}, \\
& \mathfrak{B}_{\text {aut }}^{P \wedge_{I} C}=\left\{\left(w_{1}+w_{2}, w_{1}, w_{2}\right) \mid w_{1} \in \mathfrak{B}_{\text {aut }}^{P}, w_{2} \in \mathfrak{B}_{\text {aut }}^{C}\right\} .
\end{aligned}
$$

If, in addition, $\mathfrak{B}^{P}=\mathfrak{B}_{\text {cont }}^{P} \oplus \mathfrak{B}_{\text {aut }}^{P}, \mathfrak{B}^{C}=\mathfrak{B}_{\text {cont }}^{C} \oplus \mathfrak{B}_{\text {aut }}^{C}$, Then $\mathfrak{B}^{P \wedge_{I} C}=\mathfrak{B}_{\text {cont }}^{P \wedge_{I} C} \oplus \mathfrak{B}_{\text {aut }}^{P \wedge_{I} C}$.

Proof. It is straightforward to verify that $\mathfrak{B}_{\text {cont }}^{P \wedge}$ is the maximal controllable behaviour, and that $\mathfrak{B}_{\text {aut }}^{P \wedge_{I} C}$ is autonomous. Let $w \in \mathfrak{B}^{P \wedge_{I} C}$. Then $w=\left(v_{1}+v_{2}, v_{1}, v_{2}\right)$, and by the decompositions of $\mathfrak{B}^{P}, \mathfrak{B}^{C}$, there exist elements $x_{1} \in \mathfrak{B}_{\text {cont }}^{P}, x_{2} \in \mathfrak{B}_{\text {aut }}^{P}$, and $y_{1} \in \mathfrak{B}_{\text {cont }}^{C}, y_{2} \in \mathfrak{B}_{\text {aut }}^{C}$ such that $v_{1}=x_{1}+x_{2}, v_{2}=y_{1}+y_{2}$. Consequently, there exists a decomposition of $w=z_{1}+z_{2}$ where $z_{1} \in\left(\mathfrak{B}_{\text {cont }}^{P}+\mathfrak{B}_{\text {cont }}^{C}\right) \times \mathfrak{B}_{\text {cont }}^{P} \times \mathfrak{B}_{\text {cont }}^{C}$ and $z_{2} \in\left(\mathfrak{B}_{\text {aut }}^{P}+\mathfrak{B}_{\text {aut }}^{C}\right) \times \mathfrak{B}_{\text {aut }}^{P} \times \mathfrak{B}_{\text {aut }}^{C}$, namely $z_{1}=\left(x_{1}+y_{1}, x_{1}, y_{1}\right), z_{2}=\left(x_{2}+y_{2}, x_{2}, y_{2}\right)$.

When $\mathfrak{B}^{P}=\mathfrak{B}_{\text {cont }}^{P} \oplus \mathfrak{B}_{\text {aut }}^{P}, \mathfrak{B}^{C}=\mathfrak{B}_{\text {cont }}^{C} \oplus \mathfrak{B}_{\text {aut }}^{C}$, the existence for each of $x_{1}, x_{2}, y_{1}, y_{2}$ and $z_{1}, z_{1}$ is unique. Hence $\mathfrak{B}^{P{ }_{I} C}=\mathfrak{B}_{\text {cont }}^{P{ }_{I} C} \oplus \mathfrak{B}_{\text {aut }}^{P \wedge_{I} C}$.

The following key results relate a condition of stability of a particular half-line projection to stability of the entire system behaviour.

Lemma 7.4 Let $\mathfrak{B}^{P{ }_{I} C}$ be well-posed and let

$$
\hat{\mathfrak{B}}=\left\{w=\left(w_{0}, w_{1}, w_{2}\right) \in \mathfrak{B}_{\mathrm{cont}}^{P \wedge_{I} C}:\left.w_{0}\right|_{[0, \infty)}=0\right\} .
$$

Suppose $X=\mathcal{G}_{\mathfrak{B}^{P}} \oplus \mathcal{G}_{\mathfrak{B}^{C}}$. Then for any $\left(w_{0}, w_{1}, w_{2}\right) \in \hat{\mathfrak{B}},\left.w_{1}\right|_{[0, \infty)},\left.w_{2}\right|_{[0, \infty)} \in X$. 
Proof. Let $w=\left(w_{0}, w_{1}, w_{2}\right) \in \hat{\mathfrak{B}}$. By controllability, there exist $\tau \geq 0, \bar{w}=\left(\bar{w}_{0}, \bar{w}_{1}, \bar{w}_{2}\right) \in$ $\mathfrak{B}^{P \wedge_{I} C}$ such that $\left.\bar{w}\right|_{(-\infty,-\tau]}=0$ and $\bar{w}_{[0, \infty)}=\left.w\right|_{[0, \infty)}$. Therefore $\sigma_{\tau} \bar{w}_{1} \in \mathcal{Z}_{\mathfrak{B}^{P}}, \sigma_{\tau} \bar{w}_{2} \in \mathcal{Z}_{\mathfrak{B}^{C}}$ and $\left.\bar{w}_{0}\right|_{\mathbb{R} \backslash(-\tau, 0]}=0$, and hence $\left.\bar{w}_{0}\right|_{[0, \infty)},\left.\sigma_{\tau} \bar{w}_{0}\right|_{[0, \infty)} \in X$. Since $X=\mathcal{G}_{\mathfrak{B}^{P}} \oplus \mathcal{G}_{\mathfrak{B}^{C}},\left.\sigma_{\tau} \bar{w}_{0}\right|_{[0, \infty)}=z_{1}+z_{2}$ for some $z_{1} \in \mathcal{G}_{\mathfrak{B}^{P}}, z_{2} \in \mathcal{G}_{\mathfrak{B}^{C}}$. By the well-posedness assumption, it follows that $z_{1}=\sigma_{\tau} \bar{w}_{1}, z_{2}=$ $\sigma_{\tau} \bar{w}_{2}$. Since $\bar{w}_{[0, \infty)}=\left.w\right|_{[0, \infty)}$ and $z_{1}, z_{2} \in X$, we see $\left.w_{1}\right|_{[0, \infty)},\left.w_{2}\right|_{[0, \infty)} \in X$.

Proposition 7.5 Let $\mathfrak{B}^{P}$, $B^{C}$ be linear, shift invariant behaviours with finite memory and $\mathfrak{B}^{P}=\mathfrak{B}_{\text {cont }}^{P}+\mathfrak{B}_{\text {aut }}^{P}, \mathfrak{B}^{C}=\mathfrak{B}_{\text {cont }}^{C}+\mathfrak{B}_{\text {aut }}^{C}$. Suppose $\mathfrak{B}^{P}$ and $\mathfrak{B}^{C}$ are soundly stabilizable and $\mathfrak{B}^{P \wedge} \wedge_{I} C$ is well posed. Suppose further that $X=\mathcal{G}_{\mathfrak{B}^{P}} \oplus \mathcal{G}_{\mathfrak{B}^{C}}$. Then $\mathfrak{B}^{P{ }_{I} C}$ is stable.

Proof. Suppose $w \in \mathfrak{B}^{P \wedge_{I} C}$, and $w=\left(w_{0}, w_{1}, w_{2}\right)$. We have to show if $\left.w_{0}\right|_{[0, \infty)} \in X$ then $\left.w_{1}\right|_{[0, \infty)},\left.w_{2}\right|_{[0, \infty)} \in X$. Let $w_{0} \in X$. Since $\mathfrak{B}^{P \wedge_{I} C}=\mathfrak{B}_{\text {cont }}^{P \wedge_{I} C}+\mathfrak{B}_{\text {aut }}^{P \wedge_{I} C}$, it follows that $w=x+y$, where $x=\left(x_{0}, x_{1}, x_{2}\right) \in \mathfrak{B}_{\text {cont }}^{P \wedge_{I} C}$ and $y=\left(y_{0}, y_{1}, y_{2}\right) \in \mathfrak{B}_{\text {aut }}^{P \wedge_{I} C}$. By stability of $\mathfrak{B}_{\text {aut }}^{P}, \mathfrak{B}_{\text {aut }}^{C}$ we know $\left.x_{0}\right|_{[0, \infty)}=w_{0}-\left.y_{0}\right|_{[0, \infty)} \in X$.

Let

$$
\tilde{x}_{0}= \begin{cases}0, & t \leq 0 \\ x_{0}, & t>0\end{cases}
$$

Then $\left.\tilde{x}_{0}\right|_{[0, \infty)} \in X$. Since $X=\mathcal{G}_{\mathfrak{B}^{P}} \oplus \mathcal{G}_{\mathfrak{B}^{C}}$, we see

$$
\left.\tilde{x}_{0}\right|_{[0, \infty)}=\left.\tilde{x}_{1}\right|_{[0, \infty)}+\left.\tilde{x}_{2}\right|_{[0, \infty)} \text { for some } \tilde{x}_{1} \in \mathcal{G}_{\mathfrak{B}^{P}}, \tilde{x}_{2} \in \mathcal{G}_{\mathfrak{B}^{C}} .
$$

Consider $v=\left(v_{0}, v_{1}, v_{2}\right):=\left(x_{0}-\tilde{x}_{0}, x_{1}-\tilde{x}_{1}, x_{2}-\tilde{x}_{2}\right)$. Since $x_{1}, \tilde{x}_{1} \in \mathfrak{B}_{\text {cont }}^{P}, x_{2}, \tilde{x}_{2} \in \mathfrak{B}_{\text {cont }}^{C}$, we see $x_{1}-\tilde{x}_{1} \in \mathfrak{B}_{\text {cont }}^{P}, x_{2}-\tilde{x}_{2} \in \mathfrak{B}_{\text {cont }}^{C}$ and hence $v \in \mathfrak{B}_{\text {cont }}^{P \wedge_{I} C}$. Since $\left.v_{0}\right|_{[0, \infty)}=0, v \in \hat{\mathfrak{B}}$. By the above lemma, $\left.v_{1}\right|_{[0, \infty)},\left.v_{2}\right|_{[0, \infty)} \in X$. Since $\tilde{x}_{1}, \tilde{x}_{2} \in X$, we see $\left.x_{1}\right|_{[0, \infty)},\left.x_{2}\right|_{[0, \infty)} \in X$. By the stability assumption of $\mathfrak{B}_{\text {aut }}^{P}$ and $\mathfrak{B}_{\text {aut }}^{C}$, it follows $\left.w_{1}\right|_{[0, \infty)},\left.w_{2}\right|_{[0, \infty)} \in X$. This completes the proof.

We can now give the proof of the main robust stability result. Before giving the proof we remark that the result follows straightforwardly from Proposition 7.5 once it has been shown that $X=\mathcal{G}_{\mathfrak{B}_{\text {cont }}^{P}} \oplus \mathcal{G}_{\mathfrak{B}_{\text {cont }}^{C}}$, and that this classical condition is obtained directly using the technique of [1]: we have included this part of the proof from [1] for completeness.

Theorem 7.6 Suppose $\mathfrak{B}^{P}, \mathfrak{B}^{P_{1}}, B^{C}$ are linear, shift invariant behaviours with finite memory. If:

1. $\mathfrak{B}^{P}, \mathfrak{B}^{C}$ are soundly stabilizable,

2. $\mathfrak{B}^{P \wedge{ }_{I} C}$ is well-posed, causal and uniformly stable,

3. $\mathfrak{B}^{P_{1} \wedge \wedge_{I} C}$ is well-posed, causal, and,

4. $\vec{\delta}\left(\mathfrak{B}^{P}, \mathfrak{B}^{P_{1}}\right)\left\|\Pi_{P / / C}\right\|<1$,

then $\mathfrak{B}^{P_{1} \wedge_{I} C}$ is uniformly stable.

Proof. Condition 4 implies that there exists a stable $\mathfrak{B}_{\text {aut }}^{P_{1}}$ such that $\mathfrak{B}^{P_{1}}=\mathfrak{B}_{\text {cont }}^{P_{1}}+\mathfrak{B}_{\text {aut }}^{P_{1}}$ by definition of the gap and since $\left\|\Pi_{P / / C}\right\| \geq 1$.

By condition 4 , there exists a surjective mapping $\Phi: D \subset \mathcal{G}_{\mathfrak{B}^{P}} \rightarrow \mathcal{G}_{\mathfrak{B}^{P_{1}}}$ such

$$
\|\Phi-I\|\left\|\Pi_{P / / C}\right\|<1 .
$$

Let $\left(w_{0}, w_{1}, w_{2}\right) \in \mathfrak{B}^{P_{1} \wedge_{I} C}$ with $\left.\left(w_{0}, w_{1}, w_{2}\right)\right|_{\mathcal{T}_{-}}=0,\left.\left(w_{0}, w_{1}, w_{2}\right)\right|_{\mathcal{T}_{+}} \in X$. By definition of the extended graph, $w_{1} \in \mathcal{Z}_{\mathfrak{B}^{P_{1}}}, w_{2} \in \mathcal{Z}_{\mathfrak{B}^{C}}$. 
For any $\tau>0$, by sound stabilizability and Lemma 4.7 , there exist $\bar{w}_{1} \in \mathfrak{B}^{P_{1}}, \bar{w}_{2} \in \mathfrak{B}^{C}$ such that $\left.\bar{w}_{i}\right|_{\mathcal{T}_{-}}=\left.\sigma_{\tau} w_{i}\right|_{\mathcal{T}_{-}},\left.\bar{w}_{i}\right|_{\mathcal{T}_{+}} \in X$ for $i=1,2$. This shows $\left.w_{i}\right|_{(-\infty, \tau]}=\left.w_{i}^{\tau}\right|_{(-\infty, \tau]}$ where $w_{i}^{\tau}=\sigma_{-\tau} \bar{w}_{i}$, and $\left.w_{i}^{\tau}\right|_{\mathcal{T}_{-}}=0,\left.w_{i}^{\tau}\right|_{\mathcal{T}_{+}} \in X$. By shift invariance, $\left.w_{1}^{\tau}\right|_{\mathcal{T}_{+}} \in \mathcal{G}_{\mathfrak{B}^{P}},\left.w_{2}^{\tau}\right|_{\mathcal{T}_{+}} \in \mathcal{G}_{\mathfrak{B}^{C}}$ and $T_{\tau} w_{i}=T_{\tau} w_{i}^{\tau}$. Since $\Phi$ is surjective from $\mathcal{G}_{\mathfrak{B}^{P}}$ to $\mathcal{G}_{\mathfrak{B}^{P} 1}$, there exists $w_{3}^{\tau} \in \mathcal{G}_{\mathfrak{B}^{P}}$ and $\left.w_{1}^{\tau}\right|_{\mathcal{T}_{+}}=\Phi w_{3}^{\tau}$. Write $x_{\tau}=w_{3}^{\tau}+w_{2}^{\tau} \mid \mathcal{T}_{+}$. Then by condition $2, \Pi_{P / / C} x_{\tau}=w_{3}^{\tau} \in X, \Pi_{C / / P} x_{\tau}=w_{2}^{\tau} \mid \mathcal{I}_{+} \in X$ and

$$
\begin{aligned}
T_{\tau}\left(\left.w_{0}\right|_{\mathcal{T}_{+}}\right) & =T_{\tau}\left(w_{1} \mid \mathcal{T}_{+}\right)+T_{\tau}\left(w_{2} \mid \mathcal{T}_{+}\right)=T_{\tau}\left(\left.w_{1}^{\tau}\right|_{\mathcal{T}_{+}}\right)+T_{\tau}\left(w_{2}^{\tau} \mid \mathcal{T}_{+}\right)=T_{\tau} \Phi w_{3}^{\tau}+T_{\tau}\left(w_{2}^{\tau} \mid \mathcal{T}_{+}\right) \\
& =T_{\tau} \Phi \Pi_{P / / C} x_{\tau}+T_{\tau} \Pi_{C / / P} x_{\tau}=T_{\tau} \Phi \Pi_{P / / C} T_{\tau} x_{\tau}+T_{\tau} \Pi_{C / / P} T_{\tau} x_{\tau} \\
& =T_{\tau}(\Phi-I) \Pi_{P / / C} T_{\tau} x_{\tau}+T_{\tau} x_{\tau}
\end{aligned}
$$

and

$$
T_{\tau} \Pi_{P_{1} / / C}\left(w_{0} \mid \mathcal{T}_{+}\right)=T_{\tau}\left(w_{1} \mid \mathcal{T}_{+}\right)=T_{\tau} \Phi w_{3}^{\tau}=T_{\tau} \Phi \Pi_{P / / C} x_{\tau}=T_{\tau} \Phi \Pi_{P / / C} T_{\tau} x_{\tau} .
$$

By (7.26), we have

$$
\left\|T_{\tau} x_{\tau}\right\| \leq\left\|T_{\tau}\left(w_{0} \mid \mathcal{T}_{+}\right)\right\|+\left\|T_{\tau}(\Phi-I) \Pi_{P / / C} T_{\tau} x_{\tau}\right\| \leq\left\|\left.w_{0}\right|_{\mathcal{T}_{+}}\right\|+\|\Phi-I\|\left\|\Pi_{P / / C}\right\|\left\|T_{\tau} x_{\tau}\right\|,
$$

which gives

$$
\left\|T_{\tau} x_{\tau}\right\| \leq \frac{\left\|\left.w_{0}\right|_{\mathcal{T}_{+}}\right\|}{1-\|\Phi-I\|\left\|\Pi_{P / / C}\right\|}
$$

By (7.27), we have

$$
\begin{aligned}
\left\|T_{\tau} \Pi_{P_{1} / / C}\left(w_{0} \mid \mathcal{T}_{+}\right)\right\| & \leq\left\|T_{\tau}(\Phi-I) \Pi_{P / / C} T_{\tau} x_{\tau}\right\|+\left\|T_{\tau} \Phi \Pi_{P / / C} T_{\tau} x_{\tau}\right\| \\
& \leq(1+\|\Phi-I\|)\left\|\Pi_{P / / C}\right\| \frac{\left\|w_{0} \mid \mathcal{T}_{+}\right\|}{1-\|\Phi-I\|\left\|\Pi_{P / / C}\right\|} .
\end{aligned}
$$

Hence $w_{1}=\Pi_{P_{1} / / C}\left(w_{0} \mid \mathcal{T}_{+}\right) \in X$ and therefore, by (5.15), $w_{2}=\Pi_{C / / P_{1}}\left(w_{0}\right) \in X$.

So, for any $w_{0}$ with $\left.w_{0}\right|_{\mathcal{T}_{+}} \in X,\left.w_{0}\right|_{\mathcal{T}_{-}}=0$, we have shown there exists $w_{1} \in \mathcal{G}_{\mathfrak{B}^{P_{1}}}, w_{2} \in \mathcal{G}_{\mathfrak{B}^{C}}$ such that $\left.w_{0}\right|_{\mathcal{T}_{+}}=w_{1}+w_{2}$, i.e. $X=\mathcal{G}_{\mathfrak{B}^{P_{1}}}+\mathcal{G}_{\mathfrak{B}^{C}}$. By the well-posedness assumption, the sum is direct. Applying Proposition 7.5, we see that $\mathfrak{B}^{P_{1} \wedge_{I} C}$ is stable. The above proof shows that both $\Pi_{P_{1} / / C}$ and $\Pi_{C / / P_{1}}$ are bounded. Hence $\mathfrak{B}^{P_{1} \wedge_{I} C}$ is uniformly stable.

Due to Proposition 4.9, we have

Corollary 7.7 Suppose $\mathfrak{B}^{P}, \mathfrak{B}^{P_{1}}, B^{C}$ are linear, shift invariant differential behaviours with finite memory. If $\mathfrak{B}^{P}, \mathfrak{B}^{C}$ are stabilizable, $\mathfrak{B}^{P \wedge_{I} C}$ is uniformly stable, $\mathfrak{B}^{P_{1} \wedge_{I} C}$ is well-posed, causal and $\vec{\delta}\left(\mathfrak{B}^{P}, \mathfrak{B}^{P_{1}}\right)\left\|\Pi_{P / / C}\right\|<1$, then $\mathfrak{B}^{P_{1} \wedge_{I} C}$ is uniformly stable.

\section{Relation to the behavioural $\mathcal{H}^{\infty}$ synthesis of Trentelman and Willems}

Within the context of $L^{2}$ signal spaces, classical $\mathcal{H}^{\infty}$ synthesis [21] provides constructions for controllers $C$ which achieve $\left\|\Pi_{P / / C}\right\| \leq 1$, i.e. solve the normalized version of the inequality required in our robustness theorems. The classical gap robustness results then provide an explicit description of plant uncertainties tolerated in the closed loop. In direct counterpart, and in the interests of a self-contained behavioural theory, it is relevant to relate the results of this paper to the behavioural approach to $\mathcal{H}^{\infty}$ synthesis found in [12, 17], for then our basic robust stability theorem completes a 'behavioural robust control theory' by providing an explicit robustness interpretation of the behavioural $\mathcal{H}^{\infty}$ synthesis results. 
Therefore we explicitly describe the relationship between the problem formulation of $[12,17]$ and this paper. We first consider Proposition 1 of [12]. By choosing the exogenous variable $d$ to be $w_{0}$, and the endogenous 'to be controlled' variable $f$ to be $w_{1}$, we have

$$
\mathcal{K}=\left\{\left(w_{0}, w_{1}\right) \in C^{\infty} \mid \exists w_{2} \in C^{\infty} \text { s.t. }\left(w_{0}, w_{1}, w_{2}\right) \in \mathfrak{B}^{P \wedge_{I} C}\right\},
$$

and $G_{w_{0} \rightarrow w_{1}}$ is the transfer function corresponding to $\Pi_{P / / C}$. Proposition 1 asserts that if $\mathfrak{B}^{P}, \mathfrak{B}^{C}$ are smooth differential behaviours and $\mathfrak{B}^{P \wedge_{I} C}$ is controllable then the following are equivalent:

1. In $\mathcal{K}, w_{0}$ is the input, $w_{1}$ is the output and $\left\|G_{w_{0} \rightarrow w_{1}}\right\|_{\mathcal{H}_{\infty}} \leq 1$;

2. $\mathcal{K}$ is $\Sigma$-dissipative on $\mathbb{R}_{-}$and $m(\mathcal{K})=\sigma_{+}(\Sigma)$;

3. $\left\|w_{1}\right\|_{\mathcal{L}^{2}\left(\mathbb{R}, \mathbb{R}^{f}\right)} \leq\left\|w_{0}\right\|_{\mathcal{L}^{2}\left(\mathbb{R}, \mathbb{R}^{d}\right)}, w_{0}$ is free in $\mathcal{K}$ and $\left(0, w_{1}\right) \in \mathcal{K}$ implies that $\lim _{t \rightarrow \infty} w_{1}(t)=0$,

where $\Sigma=\operatorname{diag}\left(I_{d},-I_{f}\right), \sigma_{+}(\Sigma)$ is the number of positive eigenvalues of $\Sigma$ and $m(\mathcal{K})$ is the number of "free" input variables: in the context of this paper the free variables enter additively in both the input and output channels, hence $m(\mathcal{K})=\operatorname{dim}(\mathcal{U} \times \mathcal{Y})$. We refer to $[12,17]$ for the definition of $\Sigma$-dissipativity on $\mathbb{R}_{-}$.

We now relate the above stability concepts to the notion of uniform stability, within an $L^{2}$ context, as considered in this paper. Consider the following condition:

4. $\mathfrak{B}^{P \wedge_{I} C}$ is uniformly stable, and $\left\|\Pi_{P / / C}\right\|_{L^{2}\left(\mathbb{R}_{+}\right)} \leq 1$.

Then:

Proposition 8.1 Let $X=L^{2}\left(\mathbb{R}_{+}\right)$. Suppose $\mathfrak{B}^{p}, \mathfrak{B}^{C}$ are differential behaviours and $\mathfrak{B}^{P \wedge_{I} C}$ is controllable. Then 1,2,3 and 4 are equivalent.

Proof. Proposition 1 of [12] establishes the equivalence between 1,2 and 3. It is well known that $\left\|\Pi_{P / / C}\right\|_{L^{2}\left(\mathbb{R}_{+}\right)}=\left\|G_{w_{0} \rightarrow w_{1}}\right\|_{\mathcal{H}_{\infty}}$. Hence 4. implies 1. On the other hand, suppose 1. holds. Consider $\left(w_{0}, w_{1}, w_{2}\right) \in \mathfrak{B}^{P \wedge_{I} C}$, and suppose $\left.w_{0}\right|_{\mathbb{R}_{+}} \in X$. Let $w_{0}=x_{0}+y_{0}$ where $\left.x_{0}\right|_{\mathbb{R}_{+}}=0$, $\left.y_{0}\right|_{\mathbb{R}_{-}}=0$. Then there exists $x_{1}, x_{2}, y_{1}, y_{2}$ such that $\left(x_{0}, x_{1}, x_{2}\right) \in \widehat{\mathfrak{B}^{P \wedge_{I} C}} \subset \mathfrak{B}^{P \wedge_{I} C},\left(y_{0}, y_{1}, y_{2}\right) \in$ $\mathfrak{B}^{P \wedge_{I} C}$ and $x_{1}+y_{1}=w_{1}, x_{2}+y_{2}=w_{2}$. By Lemma 7.4 , and since $\left.x_{0}\right|_{\mathbb{R}_{+}}=0 \in X$, it follows that $\left.x_{1}\right|_{\mathbb{R}_{+}}, x_{2} \mid \mathbb{R}_{+} \in X$. Since $G_{w_{0} \rightarrow w_{1}} \in \mathcal{H}_{\infty}$, and $\left.y_{0}\right|_{\mathbb{R}_{+}} \in X$ it follows that $\left.y_{1}\right|_{\mathbb{R}_{+}},\left.y_{2}\right|_{\mathbb{R}_{+}} \in X$. Hence $\left.w_{1}\right|_{\mathbb{R}_{+}}, w_{2} \mid \mathbb{R}_{+} \in X$ and consequently $\mathfrak{B}^{P \wedge_{I} C}$ is stable. The inequality in 1 . implies the inequality in 4 , hence 1 . implies 4 . as required.

Within the context of disturbance attenuation for linear controllable differential systems in an $L^{2}$ setting, the results of $[12,17]$ establish conditions under which there exists a controllable differentiable behaviour $\mathfrak{B}^{C}$ which renders $\Sigma$-dissipativity on $\mathbb{R}_{-}$of the closed loop interconnection $\mathfrak{B}^{P \wedge_{I} C}$. Here $\mathfrak{B}^{P}$ is also required to be a controllable differential behaviour. Since the resulting interconnection $\mathfrak{B}^{P \wedge_{I} C}$ is controllable, and by the above, it follows that this synthesis yields the uniform stability condition 4. above, and in turn, the robust stability theorem 7.6 provides an explicit description of a set of plants for which stability can be guaranteed.

It is worth noting that it is observed in [17] that the synthesis can be extended in an ad-hoc manner from the controllable case to the general case by introducing appropriate stabilizability assumptions in the analysis. Theorem 7.6 can be utilized to achieve these observations directly. Given a (soundly) stabilizable plant behaviour $\mathfrak{B}^{P}$, follow the $\mathcal{H}^{\infty}$ synthesis to derive a controller $\mathfrak{B}^{C}$ (which is controllable) for the controllable plant sub-behaviour $\mathfrak{B}_{\text {cont }}^{P}$. Then since $\vec{\delta}\left(\mathfrak{B}_{\text {cont }}^{P}, \mathfrak{B}^{P}\right)=0$, Theorem 7.6 can be applied to establish the required uniform stability for the interconnection of the derived controller behaviour $\mathfrak{B}^{C}$ and the original plant $\mathfrak{B}^{P}$. 


\section{An Illustrative Example}

We consider an example in an $L^{\infty}$ setting. Let the behaviours for the nominal system $P$, the perturbed system $P_{1}$ and the controller $C$ be given by:

$$
\begin{gathered}
\mathfrak{B}^{P}=\left\{\left(\begin{array}{c}
u_{1} \\
v_{1} \\
x
\end{array}\right): u_{1}, v_{1}, x \in L_{e}^{\infty}(\mathbb{R}, \mathbb{R}), \dot{x}=a u_{1}+b v_{1}\right\} \\
\mathfrak{B}^{P_{1}}=\left\{\left(\begin{array}{c}
u_{1} \\
v_{1} \\
x
\end{array}\right): u_{1}, v_{1}, x \in L_{e}^{\infty}(\mathbb{R}, \mathbb{R}), \dot{x}=a \sigma_{\tau_{1}} u_{1}+b \sigma_{\tau_{2}} v_{1}\right\}
\end{gathered}
$$

and

$$
\mathfrak{B}^{C}=\left\{\left(\begin{array}{c}
c y \\
d y \\
y
\end{array}\right): y \in L_{e}^{\infty}(\mathbb{R}, \mathbb{R}),\right\},
$$

where $a, b, c, d \in \mathbb{R}$ such that $a c+b d=-1 . \mathfrak{B}^{P}, \mathfrak{B}^{P_{1}}$ and $\mathfrak{B}^{C}$ are all linear, shift invariant behaviours with finite memory. Moreover, they are all controllable so $\mathfrak{B}^{P}=\mathfrak{B}_{\text {cont }}^{P}+\{0\}, \mathfrak{B}^{P_{1}}=$ $\mathfrak{B}_{\text {cont }}^{P_{1}}+\{0\}$ and $\mathfrak{B}^{C}=\mathfrak{B}_{\text {cont }}^{C}+\{0\}$. We also have

$$
\begin{aligned}
& \mathcal{G}_{\mathfrak{B}^{P}}=\left\{\left(\begin{array}{c}
u_{1} \\
v_{1} \\
x
\end{array}\right): u_{1}, v_{1}, x \in L^{\infty}(\mathbb{R}, \mathbb{R}), \dot{x}=a u_{1}+b v_{1},\left.u_{1}\right|_{\mathbb{R}_{-}}=\left.v_{1}\right|_{\mathbb{R}_{-}}=\left.x\right|_{\mathbb{R}_{-}}=0,\right\}, \\
& \mathcal{G}_{\mathfrak{B}^{P_{1}}}=\left\{\left(\begin{array}{c}
u_{1} \\
v_{1} \\
x
\end{array}\right): u_{1}, v_{1}, x \in L^{\infty}(\mathbb{R}, \mathbb{R}), \dot{x}=a \sigma_{\tau_{1}} u_{1}+b \sigma_{\tau_{2}} v_{1},\left.u_{1}\right|_{\mathbb{R}_{-}}=\left.v_{1}\right|_{\mathbb{R}_{-}}=\left.x\right|_{\mathbb{R}_{-}}=0\right\}, \\
& \mathcal{Z}_{\mathfrak{B}^{P}}=\left\{\left(\begin{array}{c}
u_{1} \\
v_{1} \\
x
\end{array}\right): u_{1}, v_{1}, x \in L_{e}^{\infty}(\mathbb{R}, \mathbb{R}), \dot{x}=a u_{1}+b v_{1},\left.u_{1}\right|_{\mathbb{R}_{-}}=\left.v_{1}\right|_{\mathbb{R}_{-}}=\left.x\right|_{\mathbb{R}_{-}}=0,\right\}, \\
& \mathcal{Z}_{\mathfrak{B}^{P_{1}}}=\left\{\left(\begin{array}{c}
u_{1} \\
v_{1} \\
x
\end{array}\right): u_{1}, v_{1}, x \in L_{e}^{\infty}(\mathbb{R}, \mathbb{R}), \dot{x}=a \sigma_{\tau_{1}} u_{1}+b \sigma_{\tau_{2}} v_{1},\left.u_{1}\right|_{\mathbb{R}_{-}}=\left.v_{1}\right|_{\mathbb{R}_{-}}=\left.x\right|_{\mathbb{R}_{-}}=0\right\} .
\end{aligned}
$$

The parallel projection $\Pi_{P / / C}$ operator is given by

$$
\Pi_{P / / C}\left(\begin{array}{c}
u_{0} \\
v_{0} \\
y_{0}
\end{array}\right)=\left(\begin{array}{c}
u_{0}-c y_{0}+c x \\
v_{0}-d y_{0}+d x \\
x
\end{array}\right) \quad \text { for any } u_{0}, v_{0}, y_{0} \in L_{e}^{\infty}\left(\mathbb{R}_{+}, \mathbb{R}\right)
$$

where $x$ is the unique solution to the equation

$$
\dot{x}=-x+a u_{0}+b v_{0}+y_{0}, x(0)=0 .
$$

After straightforward calculation, we see $\left\|T_{\tau} x\right\| \leq\left\|T_{\tau}\left(a u_{0}+b v_{0}+y_{0}\right)\right\|$ for any $\tau>0$ and

$$
\left\|\Pi_{P / / C}\right\| \leq 1+|c|+|d|(1+|a|+|b|) .
$$

This shows that $\mathfrak{B}^{P{ }_{I} C}$ is uniformly stable.

To estimate the gap between $\mathfrak{B}^{P}$ and $\mathfrak{B}^{P_{1}}$, we let

$$
\Phi\left(\begin{array}{c}
u \\
v \\
x
\end{array}\right)=\left(\begin{array}{l}
u \\
v \\
y
\end{array}\right) \quad \text { for any }\left(\begin{array}{c}
u \\
v \\
x
\end{array}\right) \in \mathfrak{B}^{P}
$$


where

$$
\left(\begin{array}{l}
u \\
v \\
y
\end{array}\right) \in \mathfrak{B}^{P_{1}}
$$

Then,

$$
\begin{aligned}
|x(t)-y(t)| & =\left|\int_{0}^{t}(a u(s)+b v(s)) d s-\int_{0}^{t}\left(a u\left(s-\tau_{1}\right)+b v\left(t-\tau_{2}\right)\right) d s\right| \\
& \leq|a|\left|\int_{t-\tau}^{t} u(s) d s\right|+|b|\left|\int_{t-\tau}^{t} v(s) d s\right| \\
& \leq \tau_{1}|a|\|u\|+\tau_{2}|b|\|v\|
\end{aligned}
$$

and so

$$
\left\|\left.(I-\Phi)\right|_{\mathcal{G}_{\mathfrak{B}^{P}}}\right\|=\sup \left\{\frac{\left\|T_{\tau}(x-y)\right\|}{\left\|T_{\tau}(u, v, x)^{\top}\right\|}: \tau>0,\left(\begin{array}{c}
u \\
v \\
x
\end{array}\right) \in \mathcal{G}_{\mathfrak{B}^{P}},\left(\begin{array}{c}
u \\
v \\
x
\end{array}\right) \neq 0\right\} \leq \tau_{1}|a|+\tau_{2}|b| .
$$

Hence, $\vec{\delta}\left(\mathfrak{B}^{P}, \mathfrak{B}^{P_{1}}\right) \leq|a| \tau_{1}+|b| \tau_{2}$ and, by Theorem $7.6, \mathfrak{B}^{P_{1} \wedge_{I} C}$ is uniformly stable provided

$$
\left(\tau_{1}|a|+\tau_{2}|b|\right)[1+|c|+|d|(1+|a|+|b|)]<1 .
$$

By definition of uniform stability, this means:

(i) for any $u_{1}, v_{1}, x, y \in L_{e}^{\infty}(\mathbb{R}, \mathbb{R})$ with $\dot{x}=a \sigma_{\tau_{1}} u_{1}+b \sigma_{\tau_{2}} v_{1}$ on $\mathbb{R}$ and $\left.\left(u_{1}+c y\right)\right|_{\mathbb{R}_{+}},\left(v_{1}+\right.$

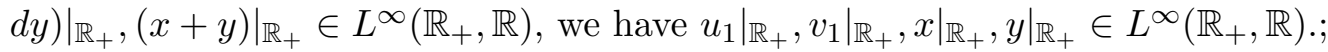

(ii) the mappings which maps $\left.\left(u_{0}, v_{0}, x_{0}\right)\right|_{\mathbb{R}_{+}}$to $\left(u_{1}, v_{1}, x_{1}\right)$ and $(c y, d y, y)$,respectively, are both bounded, where $u_{1}+c y=u_{0}, v_{1}+d y=v_{0}, x_{1}+y=x_{0},\left.(c y, d y, y)\right|_{\mathbb{R}_{-}}=0,\left.\left(u_{1}, v_{1}, x_{1}\right)\right|_{\mathbb{R}_{-}}=0$ and

$$
\dot{x}_{1}=a \sigma_{\tau_{1}} u_{1}+b \sigma_{\tau_{2}} v_{1}
$$

\section{Acknowledgements}

The authors would like to thank the reviewers and the associate editor for comments which have greatly improved the manuscript, and led to the development of the discussion in Section 6 .

\section{References}

[1] Georgiou T. and Smith M. C., Optimal robustness in the gap metric, IEEE Trans. Auto. Control, 35(1990), 673-686

[2] Georgiou T. T., On the computation of the gap metric, Systems \& Control Letters, 11(1988), $253-257$

[3] Georgiou T. and Smith M. C.,Intrinsic difficulties in using the doubly-infinte time axis for input-output control theory, IEEE Trans. Auto. Control, 40(1995), 516-518

[4] Georgiou T. and Smith M. C., Robustness analysis of nonlinear feedback systems: an input-output approach, IEEE Trans. Auto. Control, 42(1997), 1200-1221

[5] Gluesing-Luerssen H., Delay-Differential Systems with Commensurate Delays: An Algebraic Approach; Lecture Notes in Mathematics 1770; Springer 2002 
[6] Jacob, B. and Partington, J., Graphs, closability, and causality of linear time-invariant discrete-time systems. Int. J. Control, 73(2000) (11)1051-1060.

[7] Polderman J. W. and Willems J. C., Introduction to Mathematical Systems Theory, Springer, 1997

[8] Rocha, P. and Wood, J., Trajectory control and interconnection of $1 \mathrm{D}$ and $\mathrm{nD}$ systems. SIAM J. Contr. Opt., 40(2001), 107-34.

[9] Mäkilä, P.M., On three puzzles in robust control IEEE Trans. Auto. Control, 45(2000), $552-556$.

[10] Pillai, H.K. and Shankar, S., A behavioural approach to the control of distributed systems. SIAM J. Contr. Opt., 37(1998), 388-408.

[11] Sasane A. J., Distance between behaviours, Int. J. Control, 72(2003), 1214-1223

[12] Trentelman H. L. and Willems J. C., Synthesis of dissipative systems using quadratic differential forms: Part II, IEEE Trans. Auto. Control, 47(2002), 70 - 86

[13] Vinnicombe G., Uncertainty and Feedback: $\mathcal{H}_{\infty}$-shaping Control System Synthesis, Imperial College Press, London, 2001

[14] Willems J. C., From time series to linear system - Part I. Finite dimensional linear time invariant systems, Automatica, 22(1986), 561-580

[15] Willems J. C., From time series to linear system - Part II. Exact modelling, Automatica, 22(1986), 675-694

[16] Willems J. C., From time series to linear system - Part III. Approximate modelling, Automatica, 23(1987), 87-115

[17] Willems J. C. and Trentelman H. L., Synthesis of dissipative systems using quadratic differential forms: Part I, IEEE Trans. Auto. Control, 47(2002), 53-69

[18] Wood J., Rogers E. and Owens D. H., Controllable and autonomous nD linear systems, Multidimens. Systems Signal Process., 10(1999), 33-69

[19] Wood J. and Zerz E., Notes on the definition of behavioural controllability, Systems and Control Letters, 37(1999), 31-37

[20] Zames G. and El-Sakkary A. K., Unstable systems and feedback: The gap metric, Proceeding of the Allerton Conf., (Oct. 1980), 380-385

[21] Zhou, K., Doyle, J. and Glover, K. Robust and Optimal Control, Prentice Hall, 1996 